\title{
PRINCIPIOS DE UN MODELO DE DISTRIBUCIÓN Y MERCADO A PARTIR DE SRAFFA
}

\author{
Antonio Mora Plaza \\ Economista, Madrid
}

\begin{abstract}
About a fundamental model of distribution and market from Sraffa
Abstract: Sraffa make a model economic without a demand function or, simply, without demand; also without market. This article try to complete his model with a market nearly to the market theory traditional of demand with two radical situations: a competitive demand by the side and the theory monopolistic demand in the other side. In the middle all positions are possibles. A competitive market is considered if the rate profit go to zero, but the monopolistic market is different in relation to the traditional theory of demand: this market is defined so if the rate profit go to the maximum rate profit, according with the Sraffian model of single production.
\end{abstract}

Keywords: Sraffa, market, competitive, monopolistic

JEL: B24

\section{Introducción}

No hay nada en principio que pueda sostener algo parecido a una teoría de los mercados a partir de la lectura de la obra de Sraffa Producción de mercancías por medio de mercancías. No así la distribución, porque esta es el equivalente en la economía marginalista a la teoría del excedente de Sraffa y de sus ilustres predecesores clásicos, especialmente Ricardo. Menos aún algo parecido tiene Sraffa a una teoría de la asignación de los recursos como tiene el marginalismo y, más concretamente, la teoría del equilibrio general. Es verdad que no existe nada más descabellado, tanto en los fundamentos como en su contrastación empírica, que la teoría de la asignación marginalista, tanto en el equilibrio parcial de origen marshalliano como el del equilibrio general de origen walrasiano. Sin embargo, no por ello no deja de echarse de menos estas dos cosas en Sraffa: una teoría de los mercados y una teoría de la asignación de los recursos. Con este trabajo de economía matemática -donde se intenta que la economía sea el sustantivo y la matemática el adjetivo- se pretende otear en el horizonte a partir del suelo esrafiano de cuál sería los fundamentos, los pilares donde fundamentar estos temas. La razón de estas deficiencias en la teoría de los mercados y asignación de recursos en la obra del italiano se basa en dos cuestiones: Sraffa no tiene una teoría de la demanda -ni parcial ni agregada- y tampoco tiene una teoría de la producción. En principio nada impide acoplar alguna teoría de la demanda y de la producción a los fundamentos esrafianos de su teoría del excedente siempre que se respeten estos tres aspectos fundamentales de Sraffa: a) que el reparto entre rentas es frutos de la lucha de clases y no de supuestas productividades o situaciones de mercado; b) los precios deben jugar un papel de reproductor del sistema, es decir, deben ser precios de equilibrio del sistema; c) no deben ser significativos 
ni explícitos los movimientos en el margen. Ello nos lleva a la enorme dificultad de construir una teoría de los mercados en Sraffa. Mientras en el marginalismo se acepta el estudio de los mercados mediante el equilibrio parcial marshalliano de tal forma que lo que pase en un mercado no influye ni se deja influir significativamente en el conjunto de la economía, en un modelo esrafiano, en cambio, ha de compaginarse el mercado parcial con la reproducción del sistema ${ }^{1}$.

Vamos a concretar un posible modelo de mercado a partir de Sraffa dividiendo toda la economía en dos sectores, dos casos extremos, en el que uno fuera no competitivo (monopolios, oligopolios, competencia monopolística) y otro fuera de competencia perfecta. Las dos características de de estos mercados serían como sigue: 1) Los precios no jugarían ningún papel ${ }^{2}$ ni en la distribución ni en la asignación de recursos; 2) El nuevo rol del mercado en un modelo de origen esrafiano lo tendrían las tasas de ganancia y las tasas máxima de ganancia; 3) En estos mercados esrafianos, un mercado o un sector de mercancías tendería al monopolio si la tasa de ganancia tendiera a su tasa máxima de ganancia; en el otro extremo, un sector (una mercancía) tendería a la competencia perfecta si la tasa de ganancia se acercara a cero; 4) Entre estos extremos no hay una separación tajante, sino un trade off, un paso suave entre unos y otros a través de las variaciones de sus tasa de ganancia, tasas que se comportarían de acuerdo con lo enunciado en el punto (3). Un modelo económico -una explicación, unos fundamentos- que permitiera esto tendría una gran ventaja sobre los modelos de división escolástica que tiene el marginalismo de origen marshalliano. La desventaja es que ya no nos podemos mantener asidos al simple modelo de Sraffa y debemos despegarnos de su suelo de tal forma que, sin perder su soplo inspirador, podamos complementar sus fundamentos con alguna teoría del consumo y/o de la inversión y/o de la producción.

Todo lo anterior sería insuficiente si no tomamos de la realidad y de las teorizaciones de economistas, en este caso tanto clásicos como marginalistas, ricardianos como marshallianos, marxistas como walrasianos, la idea de que es la competencia entre empresas de un mismo sector -incluso competencia entre sectores por los aspectos de sustitución- la que lleva a que unos sectores y/o bienes sean o estén llevando a la venta sus productos a unos precios cercanos o no a los costes. El marginalismo no ha inventado eso, sino todo lo contrario, pero los marginalistas han tenido la astucia, suerte o visión de formalizarlo con elegancia matemática. Eso sí, con conceptualización económica descabellada como es el de la asignación de los que ellos llaman factores de acuerdo con supuestas leyes de productividad marginal; también irreal -aunque no tan

\footnotetext{
${ }^{1}$ Releyendo al día siguiente de escribir esto la biografía de Alessandro Roncaglia sobre Sraffa encontré estas palabras del gran estudioso de la historia del análisis económico: "Among other things, too direct a link between the two lines of analisis, such as the attemp to simultaneously enclose both them within the boundaries o a single mathemantical model, would have the effect of limiting the horizon of study of the barriers to entry to the the determination of sectoral profit rate differentials", pág. 153 de Piero Sraffa, edit Palgrave Macmillan, 2009. La cosa me llenó de satisfacción, porque aunque señala sólo un aspecto de la dificultad de llegar a mercados competitivos (las barreras de entrada), habla en cambio del problema principal que es el de atender a dos amos: el del cerrar el sistema ("simultaneously enclose") y el del efecto limitante de estas barreras ("effect of limiting").

${ }^{2}$ A diferencia de la visión de A. Roncaglia en Sraffa and the Theory of Prices.
} 
descabellado- el supuesto comportamiento empresarial de decidir el nivel de producción en función de la igualación de los costes marginales a los ingresos marginales. Hay que suponer en este modelo que el acercamiento de los precios a los costes en función de la competencia está implícito y que esa es la fuerza sustancial que diferencia los grados de competitividad entre sectores, empresas, zonas, etc. Debemos recabar los no marginalistas de la tradición del análisis económico que la importancia de la competencia está en la tradición clásica y claramente en Adam Smith, uno de los tópicos sobre los que gira su obra de 1776 y quizá el más importante, siendo el otro la división del trabajo. Por ello la importancia de la competencia debe -en mi opinión- incorporarse al núcleo duro de un supuesto desarrollo del análisis económico futuro de origen esrafiano por más que no aparezca implícita en la obra del italiano. Y sin embargo no le es extraño, sino todo lo contrario, porque las razones por las que Sraffa trabaje con tasas de ganancia de salario y de ganancia unitarias es doble: 1) porque su teoría analiza tendencias a largo plazo; 2) porque se mueve bajo la presión de la competencia. La diferencia entre los modelos marginalistas y los posibles de origen esrafiano no está pues en el papel de la competencia, sino en la variable que recoge este acercamiento entre precios y costes. Para el marginalismo son los precios; para los posibles modelos esrafianos son las tasas de ganancia y su acercamiento o no a las tasas máximas de ganancia, que son, como se ha demostrado, una medida del excedente relativo, que es una variable no monetaria. Si a ello le añadimos que los salarios y ganancias están en una relación no determinada por el modelo sino por la lucha de clases, todo ello da unas posibilidades y matices en las fuerzas que se mueven en lo que entendemos por espacio económico -en Sraffa el excedente, su reparto y sus límites- inimaginables para los modelos determinísticos marginalistas, modelos que llegan al extremo de determinar precios y rentas como si las clases sociales, los conflictos de clase no existieran, incluso entre sectores, empresarios grandes y pequeños, entre sectores financieros y no financieros, como si tampoco existieran o no jugaran ningún papel las instituciones y el sector público. Sólo por este hecho el marginalismo debiera estar en el cementerio de la historia del análisis económico como una curiosidad, un lamentable error, una deriva ideológica, un disparate absoluto, a pesar de la inteligencia de quienes lo inventaron y lo defendieron. Que aún perdure como núcleo duro de los estudios de microeconomía, como fundamentos del análisis económico, es una vergüenza y un caso evidente de asignación ineficiente de los recursos en la educación.

\section{El lado de la oferta}

Veamos una ecuación que podría caracterizar una economía esrafiana de las características definidas en los puntos $a, b$ y $c$. Sería como sigue (1):

$$
P_{N} Y_{N}+P_{C} Y_{C}=L_{N} W_{N}\left(I+G_{N}\right)+L_{C} W_{C}\left(I+G_{C}\right)+P_{N} X_{N}\left(I+G_{N}\right)+P_{C} X_{C}\left(I+G_{C}\right)
$$

donde los sectores no competitivos están representado por un vector de precios $\boldsymbol{P}_{\mathrm{N}}$ e inputs de trabajo $\boldsymbol{L}_{\mathrm{N}}$ de dimensión $1 \times \boldsymbol{u}$; los productos $\boldsymbol{Y}_{\mathrm{N}}$, los 
medios de producción $\boldsymbol{X}_{N}$ y las tasas de salarios $\boldsymbol{W}_{N}$ de estos sectores son matrices de dimensión uxn; por último las tasas de ganancia $\boldsymbol{G}_{N}$ tienen dimensión $\boldsymbol{n} \times \boldsymbol{n}$. Análogamente, los competitivos tienen precios $\boldsymbol{P}_{\mathrm{C}}$ e inputs de trabajo $\boldsymbol{L}_{\mathrm{C}}$ de dimensión $\mathbf{1} \times(\boldsymbol{m}-\boldsymbol{u})$; productos $\boldsymbol{Y}_{\mathrm{C}}$, medios de producción $\boldsymbol{X}_{\mathrm{C}}$ y tasas de salario $\boldsymbol{W}_{\mathrm{C}}$ son de dimensión $(\boldsymbol{m}-\boldsymbol{u}) \times \boldsymbol{n}$; y, por último, la matriz de tasa de ganancia $G_{c}$ es de dimensión $n \times n$. La ecuación (1) sería la de definición del sistema, donde hemos avanzado en varios aspectos respecto a Sraffa: a) tenemos matrices de salarios y ganancias en lugar de sendas únicas tasas de ganancia y salario; b) se ha diferenciado entre sectores competitivos y no competitivos; c) los salarios se pagan pre-factum y no post-factum (Sraffa), con lo que la tasa de ganancia gira sobre todos los costes, incluyendo en estos a los salariales; d) las matrices de productos y medios de producción no son cuadradas, con lo cual evitamos el supuesto irreal de que el número de productos finales distintos $(\boldsymbol{m})$ sean iguales al de medios de producción distintos $(\boldsymbol{n})$ (independientemente de las cantidades empleadas y producidas).

Vamos ahora a trabajar con las tasas máximas de ganancia. Estas son el equivalente a la razón-patrón de la producción simple esrafiana para la producción conjunta, que es en la que estamos. En efecto, lejos queda el paraíso de Sraffa de la producción simple en la que la matriz de productos finales $Y$ era cuadrada y diagonal (con ceros en los elementos que no están en la diagonal principal) y cuyo rango coincidía con la de los medios de producción $\boldsymbol{X}$. Entonces, si además $\boldsymbol{A}$ era no negativa e indescomponible, se podía aplicar el teorema de Rouché-Frobenius a la ecuación transformada de definición del sistema $\boldsymbol{P}=(\mathbf{1}+\boldsymbol{R}) \boldsymbol{P A}$, siendo $\boldsymbol{A}=\boldsymbol{X} \boldsymbol{Y}^{-1}$ y $\boldsymbol{R}$ la famosa razón-patrón. Si además la matriz $\boldsymbol{A}$ de requerimientos era productiva ello nos aseguraba un vector de precios estrictamente positivos. Ahora ese paraíso se ha perdido: $\boldsymbol{Y}$ no es cuadrada, no es diagonal y el modelo intenta reflejar una economía donde las formas que adoptan los mercados en relación a la demanda va desde la competencia perfecta al monopolio. Por supuesto que ya hemos definido qué entendemos por competencia perfecta y monopolística (el caso extrema). La diferencia fundamental entre el modelo marginalista de mercado y el que aquí se propone es que los precios en este no juegan ningún papel. Dicho de otra manera, cualquiera vector de precios es compatible con cualquiera de las formas de mercado de raíz esrafiana. Aquí las variables fundamentales son las tasas de ganancia. En principio tampoco lo juega las productividades marginales de los modelos marginalistas, pero este modelo de raíz esrafiana presenta una analogía con aquellas: las tasas de ganancia máximas, que son a la vez una medida del excedente y una medida de la productividad. No hay nada equivalente en los modelos marginalistas, pero estas tasas máximas son decisivas en este modelo y, me atrevería a decir, en los desarrollos esrafianos del futuro. Sin más elucubraciones presentamos la $2^{a}$ ecuación de definición del sistema donde aparecen estas tasas máximas de ganancia:

$$
P_{N} Y_{N}+P_{C} Y_{C}=P_{N} X_{N}\left(I+G_{m N}\right)+P_{C} X_{C}\left(I+G_{m C}\right)
$$

donde $\boldsymbol{G}_{\mathrm{mN}}$ y $\boldsymbol{G}_{\mathrm{mc}}$ son las tasas máximas del sector no competitivo y competitivo, respectivamente. Estas tasas máximas lo son porque acaparan todo el excedente, es decir, porque los salarios han desaparecido. Ha de 
entenderse, en todo caso y en aras del realismo, que los salarios que permiten la reproducción del sistema están comprendidos entre los propios medios de producción $\boldsymbol{X}_{\mathrm{N}}$ y $\boldsymbol{X}_{\mathrm{C}}$, como lo hace Sraffa ${ }^{3}$; también debe entenderse que estas tasas máximas representan el límite máximo de creación de excedente del sistema, que es como hay que entenderlo en el presente contexto. Del resultado de la conjunción del sistema de ecuaciones (1) y (2) surge:

$$
P_{N} X_{N}\left(G_{m N}-G_{N}\right)+P_{C} X_{C}\left(G_{m C}-G_{C}\right)=L_{N} W_{N}\left(I+G_{N}\right)+L_{C} W_{C}\left(I+G_{C}\right)
$$

En la ecuación (3) ya están los elementos que van prefigurar lo que viene. Hay que hacer notar que aquí, en esta ecuación, los productos finales, tanto de los sectores no competitivos como los de los competitivos, han desaparecido; también sus precios. El que aparezcan los mismos vectores precios premultiplicando a los medios de producción se debe a que hemos empleado el mismo vector de precios para productos y medios, pero ello no es una exigencia del modelo. Podríamos muy bien haber partido de vectores de precios diferentes, lo cual añadiría generalidad y realismo en un grado al que no se puede comparar ningún modelo marginalista; tampoco macroeconómico de raíz keynesiano/kaleckiano, porque estos modelos sufren el inconveniente de su inevitable agregación ${ }^{4}$. Aunque quizá no sea fácil de percibir, ya vemos en (3) que va a existir una relación inversa entre las tasas de ganancia -tanto las normales o exigidas por los empresarios como las máximas- del sector no competitivo con el competitivo, ¡sean cuales sean los precios y el nivel de salarios! Para ver esto con más detenimiento vamos a proceder a simplificar (3) de una manera no habitual. Lo normal es sustituir las matrices de tasas de ganancia por sendas tasas unitarias de ganancia 5 . Lo que vamos a hacer es matemáticamente igual, pero conceptualmente muy distinto, yendo siempre el concepto por delante de su formalización, tal como exige el uso adecuado del instrumental matemático en las esferas del conocimiento. Para ello vamos a plantear el siguiente sistema de ecuaciones:

$$
\begin{aligned}
& L_{N} W_{N}\left(I+G_{N}\right) I=\left(1+g_{N}\right) L_{N} W_{N} I \\
& L_{C} W_{C}\left(I+G_{C}\right) I=\left(1+g_{C}\right) L_{C} W_{C} I
\end{aligned}
$$

donde $\boldsymbol{g}_{\mathrm{N}}$ y $\boldsymbol{g}_{\mathrm{C}}$ son tasas unitarias de ganancia de ambos sectores de la economía e I es el vector de unos $n \times 1$, cuyo fin es convertir las ecuaciones matriciales en escalares mediante su agregación. No son, sin embargo, tasas unitarias cualesquiera, sino que son precisamente medias ponderadas de las

\footnotetext{
${ }^{3}$ Capítulo I de Producción de mercancías por medio de mercancías, pág. 2, ed. Oiko-Tau, 1966.

${ }^{4}$ Salvo que se parta de las ecuaciones de Sraffa y se apliquen al modelo keynesiano de demanda efectiva. El autor de este artículo lo ha hecho en Modelo integrado Sraffa-Keynes.

${ }^{5}$ En la obra de Kurz y Salvador Theory of Production, que es de 1995, aún parte de sendas unitarias tal y como hace Sraffa en su obra (pág. 1, edit. Cambridge University Press).
} 
matrices de tasas de ganancia $\boldsymbol{G}_{\mathrm{N}}$ y $\boldsymbol{G}_{\mathrm{C}}$, en las que los pesos de ponderación son los elementos de los vectores de las masas salariales $\boldsymbol{L}_{N} \boldsymbol{W}_{\mathrm{N}}$ y $\boldsymbol{L}_{\mathrm{C}} \boldsymbol{W}_{\mathrm{C}}$. Ello se puede comprobar despejando $\boldsymbol{g}_{\mathrm{N}}$ y $\boldsymbol{g}_{\mathrm{C}}$ definimos la tasa de salario media ponderada $\boldsymbol{w}$ :

$$
\begin{aligned}
& g_{N}=\frac{L_{N} W_{N} G_{N} I}{L_{N} W_{N} I} \\
& g_{C}=\frac{L_{C} W_{C} G_{C} I}{L_{C} W_{C} I}
\end{aligned}
$$

Hacemos estas mismas consideraciones con las tasas máximas de ganancia, es decir, convertimos las matrices de las tasas de ganancia máximas en tasas unitarias no arbitrarias, sino surgidas como medias ponderadas ${ }^{6}$. Con estas consideraciones y sustituyendo (6) y (7) en (3) queda:

$$
w=\frac{\left(1+g_{N}\right) L_{N} W_{N} I+\left(1+g_{C}\right) L_{C} W_{C} I}{\left(1+g_{N}\right) L_{N} I+\left(1+g_{C}\right) L_{C} I}
$$

Y estas tres últimas ecuaciones sustituidas en (3) da lugar a:

$$
w=\frac{\left(g_{m N}-g_{N}\right) P_{N} X_{N} I+\left(g_{m C}-g_{C}\right) P_{C} X_{C} I}{\left(1+g_{N}\right) L_{N} I+\left(1+g_{C}\right) L_{C} I}
$$

Lo sorprendente de (9) es que, a pesar de que son tasas unitarias de salario (una), de ganancias (dos) y de ganancias máximas (dos), no hay pérdida de generalidad respecto a (3) y muy bien podríamos llegar a esta ecuación ja partir de los datos de la realidad! En (9) vemos que un aumento de las tasas de ganancia de ambos sectores de la economía ( $\boldsymbol{g}_{\mathrm{N}}$ y $\boldsymbol{g}_{\mathrm{C}}$ ) provocaría una disminución de la tasa de salario; en cambio, un aumento de las tasas máximas $\left(\boldsymbol{g}_{\mathrm{N}}\right.$ y $\left.\boldsymbol{g}_{\mathrm{C}}\right)$ originarían su aumento. Esta ecuación es compatible con cualquier vector de precios, de inputs de trabajo y con cualquier matriz de medios de producción, como puede comprobarse en (9). En esta ecuación tenemos todo lo equivalente en el mundo esrafiano a la teoría marginalista de la formación de los precios y asignación de recursos. Supongamos que no hubiéramos entrado en consideraciones entre sectores competitivos y no competitivos. En este caso hipotético, la ecuación de equilibrio del sistema dado a partir de la tasa máxima de ganancia $\boldsymbol{g}_{\mathrm{m}}$ daría:

$$
w=\frac{\left(g_{m}-g\right)}{(1+g)} \times \frac{P X I}{L I}
$$

${ }^{6} P_{N} X_{N}\left(G_{m N}-G_{N}\right) I=\left(g_{m N}-g_{N}\right) P_{N} X_{N} I$

у $P_{C} X_{C}\left(G_{m C}-G_{C}\right) I=\left(g_{m C}-g_{C}\right) P_{C} X_{C} I$ 
Llegado a este punto no podemos eludir la consideración que merece la tasa máxima de ganancia y para ello nos remitimos al apéndice. La función (10) que relaciona la tasa de salario $\boldsymbol{w}$ y la tasa de ganancia $\boldsymbol{g}$ es convexa, con puntos de corte en $\boldsymbol{w}(\boldsymbol{g}=\mathbf{0})=\boldsymbol{g}_{\mathrm{m}} \boldsymbol{P X \mathbf { I }} / \boldsymbol{L I}$ y en $\boldsymbol{g}(\boldsymbol{w}=\mathbf{0})=\boldsymbol{g}_{\mathrm{m}}$. Que la relación salario-ganancia (frontera) sea una curva y no una recta se debe a que hemos establecido los salarios pre-factum y no post-factum (Sraffa). Si sustituimos en (10) el valor de la tasa máxima de ganancia de la ecuación (A8) del apéndice y tomamos como numerario $\boldsymbol{P X \mathbf { I }} / \mathbf{L I}$ queda:

$$
w=\frac{\left[f\left[X^{-1}(Y-X)\right]-g\right]}{(1+g)}
$$

Ahora podemos comparar la manera en que asigna los factores el modelo marginalista. Este viene definido por la ecuación:

$$
\frac{w}{d Y / d L}=\frac{g}{d Y / d X}=P \Rightarrow w=\frac{d Y / d L}{d Y / d X} \times g
$$

Veamos las diferencias entre (11) como medio de asignación de recursos y retribución de los factores (medios) de raíz esrafiana y (12) como modelo marginalista: 1) En el modelo esrafiano la retribución del factor (en este caso el trabajo) es proporcional al excedente; en el modelo marginalista no existe esa noción; 2) En el modelo esrafiano el excedente es también una medida de la productividad (no marginal) del sistema en su conjunto; en el marginalista la retribución de los factores sólo depende de su mercado (Marshall) y de la función de producción; 3) En el esrafiano no es exigible ninguna relación específica entre producción $\boldsymbol{Y}$ y medios de producción $\boldsymbol{X}$; en el marginalista es necesario una relación decreciente, es decir, ha de suponerse en algún momento que se da una variación del producto con rendimientos decrecientes a escala (=costes crecientes) para obtener un vector de precios de equilibrio ${ }^{7}$, lo que supone una concreción no siempre pertinente; 4) En el esrafiano la tasa de salario es siempre inversamente proporcional a la tasa de ganancia $(\boldsymbol{g})$; en el marginalista depende de la relación de productividades (12), aunque la totalidad del producto ha de repartirse entre todos los factores (condición de homogeneidad de Euler); 5) En el modelo esrafiano se toma todo el vector de precios, mientras que en el marginalista de equilibrio parcial marshalliano no. Otra cosa es si partimos de alguna noción de equilibrio general walrasiano. En este caso han de tener solución las variables monetarias (precios y retribuciones de los factores) del sistema. La ecuación (11) de origen esrafiano representa una verdadera alternativa a los modelos walrasianos de equilibrio general o de equilibrio parcial marshalliano. Ambos modelos son incompatibles y el representado por (12) hace tiempo que es un cadáver, pero un cadáver

\footnotetext{
${ }^{7}$ Si no son crecientes los costes marginales cuando se igualan al precio no hay una curva de oferta de la empresa, y si se extiende al sector, no hay una curva de oferta del sector, con lo que no se producirá la anhelada asignación óptima de los recursos entre los diferentes productos.
} 
que vive en las universidades y en el subconsciente del neoliberalismo. La ecuación (11) la podemos dejar como:

$$
w(1+g)+g=f\left(X^{-1}(Y-X)\right)
$$

En lado izquierdo son las variables monetarias de la retribución de los factores; la del lado derecho es el excedente. Obsérvese que si los salarios fueran postfactum (Sraffa) obtendríamos:

$$
w+g=f\left(X^{-1}(Y-X)\right)
$$

donde queda con evidencia meridiana cómo se reparte el excedente (relativo) ${ }^{8}$.

\section{Relación entre tasas de ganancia}

Toca ahora establecer la relación entre tasas de ganancia de un mercado competitivo y uno no competitivo de acuerdo con los criterios de diferenciación asentados como hipótesis en la introducción. De (1) se obtiene:

$$
P_{N} Y_{N} I+P_{C} Y_{C} I=\left(1+g_{N}\right) L_{N} W_{N} I+\left(1+g_{C}\right) L_{C} W_{C} I+\left(1+g_{N}\right) P_{N} X_{N} I+\left(1+g_{C}\right) P_{C} X_{C} I
$$

donde hemos pos-multiplicado (1) por el vector vertical de unos I de dimensión $n \times 1$ (para poder sumar los elementos de los vectores) y hemos sustituido las matrices de tasas de ganancia por una expresión unitaria de (en este caso, dos tasas, una por cada sector) de acuerdo con las ecuaciones (6) y (7). Ahora tomamos como numerario ${ }^{9}$ :

$$
P_{N} Y_{N} I+P_{C} Y_{C} I=1
$$

Y si despejamos la tasa de ganancia de los sectores no competitivos $\boldsymbol{g}_{\mathrm{N}}$ quedaría:

$$
g_{N}=\frac{1-\left(1+g_{C}\right)\left[L_{C} W_{C} I+P_{C} X_{C} I\right]-\left[L_{N} W_{N} I+P_{N} X_{N} I\right]}{L_{N} W_{N} I+P_{N} X_{N} I}
$$

Y (18) representa el reparto entre tasas de ganancia según que los sectores se deslicen desde la competencia a la no competencia ${ }^{10}$. De esta ecuación

\footnotetext{
${ }^{8}$ Hay que recordar que $\mathbf{f}\left(\mathbf{X}^{-1}(\mathbf{Y}-\mathbf{X})\right)$ representa la tasa máxima de ganancia, como se puede ver en el apéndice de este artículo.

${ }^{9}$ Tomar un numerario en una ecuación es dividir la ecuación entre el supuesto numerario.

10 Podíamos haber empleado una tasa unitaria de salario para los productos competitivos haciendo que $\mathbf{W}_{C} \mathbf{L}_{C} \mathbf{l}=\mathbf{w}_{C} \mathbf{L}_{C} \mathbf{l}$., siendo $\mathbf{w}_{c}$ el nuevo salario unitario del sector competitivo que es
} 
obtenemos las siguientes conclusiones no triviales: 1) La relación entre las tasas de ganancia de los sectores no competitivos $\left(\boldsymbol{g}_{\mathrm{N}}\right)$ y los competitivos $\left(\boldsymbol{g}_{\mathrm{C}}\right)$ es continua -sin brusquedades- y lineal, lo cual puede resultar sorprendente dada la complejidad formal de los supuestos de partida; 2) Esta relación lineal inversa es compatible con cualquier vector de precios que surja como solución de la ecuación de definición del sistema (1) puesto que estos precios no están presentes en (18); 3) La linealidad es más sorprendente dado que hemos pasado de los datos que expresan las matrices de salarios y ganancias a las tasas de ganancia de estas últimas. Ya vimos que la simplificación no se hacía como en el marginalismo de acuerdo con los libros más o menos de divulgación que se estudia en las universidades partiendo como hipótesis de tasas unitarias, sino que estas tasas (y también la de los salarios cuando se empleen también tasas unitarias) son medias ponderadas de los datos originales; 4) Cuando aumentan las tasas de salario disminuye la tasa de ganancia, aquí del sector no competitivo $g_{N}$; 5 ) Si en (18) se hiciera cero la tasa de ganancia del sector competitivo $\left(\boldsymbol{g}_{\mathrm{C}}\right)$, no por ello la tasa de ganancia $\boldsymbol{g}_{\mathrm{N}}$ alcanzaría la tasa máxima de ganancia, tasa que hemos puesto como criterio de sector monopolístico. Y esto porque llevar la tasa de ganancia a la máxima exigiría que los salarios se hicieran cero, cosa que no ocurre (salvo que lo supongamos) en (18). Esto exige una rectificación y una reflexión. La primera es que ya no podríamos mantener el criterio de que los monopolios tienden a la tasa máxima de ganancia porque la economía no podría reproducir su ciclo; la reflexión está relacionado con lo anterior y exige que la política económica frene la tendencia de los monopolios a aumentar sus ganancias desde el ombligo de su situación de mercado, porque lo que es factible para una mercancía (sector) por esta situación de privilegio, resulta nefasto para el conjunto de la economía si, fruto de este privilegio, los salarios disminuyeran significativamente; 6) Los precios no influyen en el reparto entre las tasas de ganancia de los dos supuestos sectores en que hemos dividido la economía. Por ello, de alguna manera, las tasas máximas de ganancia se comportan en este modelo de producción conjunta y de división del mercado como la razónpatrón de la producción simple esrafiana. En realidad la gran diferencia entre el modelo de la razón-patrón esrafiana y esta extendida y generalizada con tasas máximas de ganancia es la de que no podemos asegurar que en el proceso de reproducción del sistema podamos encontrar un vector de precios de equilibrio todos positivos, porque esto sólo se logra aplicando Rouché-Frobenius ${ }^{11}$ en la producción simple (es decir, ahí cuando se puede), que es el equivalente a los teoremas de Brower y Kakutani en los modelos de equilibrio general marginalista. Para ello tenemos la guía del maestro italiano que nos decía que entonces había que acudir al comportamiento (sociológico) del empresario que tendería a desechar los valores de las variables monetarias y empleo de medios de producción que dieran lugar a precios negativos.

una media ponderada con los inputs de trabajo de los elementos de la matriz $\mathbf{W}_{\mathrm{C}}$ de salarios. Las mismas consideraciones valdrían para el sector no competitivo.

${ }^{11} \mathrm{Y}$ suponiendo además que la matriz $\mathbf{A}$ de requerimientos se productiva, es decir, que $\mathbf{X}>\mathbf{X A}$, con $\mathbf{A}=\mathbf{X} \mathbf{Y}^{-1}$. 


\section{Incorporación de la demanda}

Damos ahora un paso más porque, a pesar de la generalidad con que hemos trabajado con los medios de producción, productos, tasas de ganancia y de salarios en relación al modelo de Sraffa de Producción de mercancías por medio de mercancías, no nos hemos movido del lado de la oferta. Dicho de otra forma, no hemos salido del excedente. A la teoría del excedente podríamos acoplarla, por ejemplo, una teoría de la producción (concretada en una función de producción) y/o una teoría de la demanda keynesiana/kaleckiana y/o una teoría de la demanda de inversión. El primer caso -una teoría de la producción- no la contemplamos por varios motivos: 1) porque eso sería ir contra el espíritu de Sraffa, aunque este motivo no tenga mucho peso; 2) porque las teorías marginalistas de la producción al uso carecen de prestigio y solvencia intelectual por su falta de realismo, empezando por la propia teoría del capital, núcleo duro de cualquier teoría del equilibrio general; 3) porque al añadir una teoría de la producción que relacionara de manera funcional la producción y sus medios reduciríamos un grado de libertad ( $\boldsymbol{n}$ grados si contemplamos $\boldsymbol{n}$ funciones, es decir, tantos como sectores) al sistema, con lo cual la relación en libertad (un grado) entre salarios y ganancias se perdería y caeríamos en las garras marginalistas de las productividades y sus supuestas retribuciones. Sin embargo, el modelo de Sraffa es compatible y complementario con una teoría de la demanda y/o de la inversión de tipo keynesiana/kaleckiana. También de una teoría de la demanda cuyo componente el consumo no dependiera de la propensión marginal del consumo, sino de una derivada de las propias rentas esrafianas. Es la que aquí se propone. La ecuación que definiría el consumo sería:

$$
C=a\left[L_{N} W_{N}+L_{C} W_{C}\right] I+b\left[\left(L_{N} W_{N}+P_{N} X_{N}\right) G_{N}+\left(L_{C} W_{C}+P_{C} X_{C}\right) G_{C}\right] I
$$

donde el vector I que aparece es el vector vertical de unos $n \times 1$. Lo que nos dice (19) es que el consumo depende de las rentas de los asalariados (primer corchete) y de las rentas no salariales (segundo corchete). No puede haber más generalidad. Es una función de consumo que simplemente parte de un hecho estadístico y natural y es el de que parte de las rentas de la producción se convierten o sirven para el consumo. Es el flujo circular de la renta. Aquí hemos sorteado la propensión al consumo keynesiano/kaleckiano, aunque esta hubiera sido una alternativa válida a la que se propone en (19). Con más razón hemos huido como de la peste de cualquier derivación del consumo proveniente de utilidades margines o curvas de indiferencia. Es (19) un tema de contrastación empírica, de toma de datos de la realidad. La demanda agregada estaría definida de la manera habitual por la suma de las demandas de consumo $\boldsymbol{C}$, la de inversión $\boldsymbol{I}_{\mathrm{K}}$, el gasto público $\boldsymbol{G}_{\mathrm{P}}$, menos los impuestos $\boldsymbol{T}$, más las exportaciones $\boldsymbol{E}_{\mathrm{X}}$ y menos las importaciones $\boldsymbol{I}_{\mathrm{M}}$. Es decir:

$$
\text { Demanda }=C+I_{K}+G_{P}-T+E_{X}-I_{M}
$$


Pues bien, si ahora sustituimos (19) en (20) e igualamos su resultado con la oferta definida en $(1)^{12}$ y tras manipulaciones algebraicas elementales se obtiene:

$$
(1-b)\left[L_{N} W_{N}+P_{N} X_{N}\right] G_{N} I+(1-b)\left[L_{C} W_{C}+P_{C} X_{C}\right] G_{C} I=
$$

$$
=I_{K}+H-\left[L_{N} W_{N}+P_{N} X_{N}\right] I-\left[L_{C} W_{C}+P_{C} X_{C}\right] I+a\left[L_{C} W_{C}+L_{N} W_{N}\right] I
$$

donde hemos llamado $\boldsymbol{H}$ a la suma del gato público $\boldsymbol{G}_{\mathrm{p}}$, menos los impuestos $\boldsymbol{T}$, más las exportaciones $\boldsymbol{E}_{\mathrm{X}}$, menos las importaciones $\boldsymbol{I}_{\mathrm{M}}$. Aunque ya se ven en (21) algunas relaciones no triviales entre sus variables, vamos a simplificar esta ecuación mediante las ecuaciones:

$$
\begin{gathered}
g_{N}=\frac{\left[L_{N} W_{N}+P_{N} X_{N}\right] G_{N} I}{\left[L_{N} W_{N}+P_{N} X_{N}\right] I} \\
g_{C}=\frac{\left[L_{C} W_{C}+P_{C} X_{C}\right] G_{C} I}{\left[L_{C} W_{C}+P_{C} X_{C}\right] I}
\end{gathered}
$$

Lo notable de (22) y (23) es que vamos a poder pasar las matrices de tasas de ganancia $\boldsymbol{G}_{N}$ y $\boldsymbol{G}_{C}$ de la ecuación (21) a tasas unitarias $\boldsymbol{g}_{N}$ y $\boldsymbol{g}_{\mathrm{C}}$ sin pérdida de generalidad, porque estas tasas son medias ponderadas de los datos de la realidad que representan esta última pareja de ecuaciones. Hecho esto y despejado la tasa de ganancia del sector no competitivo $\boldsymbol{g}_{\mathrm{N}}$ en función del competitivo $\boldsymbol{g}_{\mathrm{C}}$ queda:

$$
g_{N}=\frac{1}{(1-b)} \times\left[\frac{I_{K}+H-(1-a)\left[L_{N} W_{N}+L_{C} W_{C}\right] I-\left[P_{C} X_{C}+P_{N} X_{N}\right] I}{\left[L_{N} W_{N}+P_{N} X_{N}\right] I}\right]-\frac{b\left[L_{C} W_{C}+P_{C} X_{C}\right] I}{\left[L_{N} W_{N}+P_{N} X_{N}\right] I} \times g_{C}
$$

¡Por fin hemos obtenido la relación entre las tasas de ganancia de los sectores! De (24) surgen las siguientes conclusiones no triviales: 1) La relación, el trade off, entre las dos tasas de ganancia unitarias que representan a la economía es inversa y jlineal!, a pesar de lo complejo de su deducción; 2) El elemento autónomo de la tasa de ganancia $\boldsymbol{g}_{\mathrm{N}}$ (el primer corchete) tiene jun multiplicador! $\{\mathbf{1} /(\mathbf{1}-\boldsymbol{b})\}$ del mismo tipo que el keynesiano, y significa que la tasa de ganancia no competitiva ${ }^{13}$ aumentará si aumenta la inversión $\boldsymbol{I}_{\mathrm{K}} \mathrm{y} / \mathrm{o}$ se incurre en un déficit presupuestario $\boldsymbol{G}_{\mathrm{P}} \boldsymbol{T} \boldsymbol{T}$ y/o se produce un superávit de balanza de pagos $\boldsymbol{E}_{\mathrm{X}} \boldsymbol{I}_{\mathrm{M}}$, de forma análoga a la tradición keynesiana y en contra de las corrientes neoliberales, neoclásicas y marginalistas imperantes actualmente; 3) El intercambio entre ambas tasas no depende de la producción

\footnotetext{
${ }^{12}$ Multiplicada la ecuación (1) de definición del sistema esrafiano por el vector vertical de unos I para igualar oferta agregada con demanda agregada.

${ }^{13}$ Dado que ambos tipos de ganancia son simétricas en (21), lo mismo que se dice de una tasa de ganancia se puede decir de la otra.
} 
final $\boldsymbol{Y}_{\mathrm{N}}$ y $\boldsymbol{Y}_{\mathrm{C}}$; 4) Dicho intercambio es compatible con cualesquiera vectores de precios $\boldsymbol{P}_{\mathrm{N}}, \boldsymbol{P}_{\mathrm{C}}$ y cualesquiera matrices de salarios $\left.\boldsymbol{W}_{\mathrm{N}}, \boldsymbol{W}_{\mathrm{C}} ; 5\right)$ A pesar de estas conclusiones teorizantes, el realismo de (24) es escalofriante porque podríamos sustituir directamente los datos de la realidad y someterlo a una verificación popperiana, a diferencia de las teorías del equilibrio general que no admiten el criterio de falsibilidad; 6) No existe equilibrio automático en la economía a pesar de la igualdad entre la oferta agregada y la demanda agregada que se ha supuesto, sino que ello va a depender del volumen de las inversiones $\boldsymbol{I}_{\mathrm{K}}$, del déficit presupuestario $\boldsymbol{G}_{\mathrm{P}}-\boldsymbol{T}$ y del saldo de la balanza comercial $\boldsymbol{E}_{\mathrm{X}} \boldsymbol{I}_{\mathrm{M}}$. Esta conclusión es contraria a las concepciones neoclásicas y marginalistas que creen en que los mercados buscarán por sí solos los equilibrios que permitan reproducir el sistema sin ciclo económico ${ }^{14}$.

Resumiendo (24), podemos decir que la tasa de ganancia $\boldsymbol{g}_{\mathrm{N}}$ aumentará con el aumento de ¡la inversión $\boldsymbol{l}_{k}$ !, ¡el déficit presupuestario $\boldsymbol{G}_{\mathrm{p}}-\boldsymbol{T}$ !, el superávit exterior $\boldsymbol{E}_{\mathrm{X}} \boldsymbol{I}_{\mathrm{M}}$, jlas tasas de consumo de las rentas salariales a y las no salariales $\boldsymbol{b}$ ! (aunque de manera distinta), y disminuirá con el aumento de la otra tasa de ganancia $\boldsymbol{g}_{\mathrm{C}}$, los salarios $\boldsymbol{W}_{\mathrm{N}}, \boldsymbol{W}_{\mathrm{C}}$ y los precios $\boldsymbol{P}_{\mathrm{N}}, \boldsymbol{P}_{\mathrm{C}}$.

Puede observarse además el aspecto asimétrico del consumo derivado las propensiones a consumir de las rentas salariales $\boldsymbol{a}$ y de las no salariales $\boldsymbol{b}$, porque si la primera es lineal, la segunda es crecientemente creciente, por lo que una política económica que quisiera incidir en el aumento de la demanda agregada a través de las propensiones a consumir tendría unos efectos cuantitativamente distintos, aunque ambos crecientes. De hecho, en el modelo no establece ningún límite al aumento de a, pero no podría decirse lo mismo de $\boldsymbol{b}$, porque si esta propensión tendiera a $\mathbf{1}$, es decir, a que todas las rentas no salariales se volcaran en el consumo, las tasas de ganancia tenderían jal infinito! En aras del realismo ello no puede ser, por lo que forzosamente ha de haber una limitación de facto en esta propensión al consumo. Este comportamiento es análogo al que surge en la obra de Sraffa en su capítulo sobre las habas, es decir, el de las mercancías que se auto-reproducen ${ }^{15}$, cuyos precios tenderían al infinito si aumentara la tasa de ganancia del sistema. La razón de este comportamiento teórico está en (19), donde se puede comprobar que la propensión a consumir $\boldsymbol{b}$ de las rentas no salariales está multiplicando a las tasas de ganancia -a diferencia del componente a de las rentas salariales-, por lo que un aumento de aquellas rentas daría lugar a un aumento del consumo a través de $\boldsymbol{b}$ (los productos implícitos $\boldsymbol{b} \boldsymbol{G}_{N}$ y $\boldsymbol{b} \boldsymbol{G}_{C}$ en (19)), que a su vez aumentarían las ganancias al igualar oferta agregada y demanda agregada, es decir, al exigir la condición global de equilibrio.

En (24) también puede observarse la condición necesaria para que la tasa de ganancia de las rentas no salariales fuera mayor que cero. Según esta ecuación obtenida de la ecuación de definición del sistema (1), del

\footnotetext{
${ }^{14}$ Este es el gran error de las concepciones neoliberales, neoclásicas y marginalistas de la economía: creer que incluso cuando se da un equilibrio en los diversos mercados de la economía traerá por ello el equilibrio en toda la economía (equilibrio general) que permita la reproducción del sistema en las mismas condiciones de partida.

${ }^{15}$ Apéndice B, páginas 125 y 126 de Producción de mercancías por medio de mercancías, ediciones Oiko-Tau.
} 
comportamiento del consumo en (19) y del equilibrio al igualar oferta y demanda agregadas, la condición de tasa de ganancia positiva (o de tasas por ser $\boldsymbol{g}_{\mathrm{N}}$ una tasa surgida de medias ponderadas de (22)) es que se cumpla lo que sigue (25):

$g_{N}>0 \quad$ si $I_{k}+H>(1-a)\left[L_{N} W_{N}+L_{C} W_{C}\right]+\left[P_{C} X_{C}+P_{N} X_{N}\right] I+b\left[L_{C} W_{C}+P_{C} X_{C}\right] g_{C}$

Y (25) es también contraria a las concepciones neoliberales, neoclásicas y marginalistas de la economía, porque exige que para que las ganancias ${ }^{16}$ sean positivas, la suma de las inversiones públicas, más el déficit presupuestario, más el superávit de la balanza de pagos ha de ser mayor que el lado derecho de la inecuación, aún cuando no tengamos una interpretación económica de lo que significa este lado.

\section{Un sólo sector}

De existir un sólo sector de la economía, la ecuación (24) quedaría como:

$$
g=\frac{1}{(1-b)} \times \frac{I_{K}+\left(G_{P}-T\right)+\left(E_{X}-I_{M}\right)-(1-a) L W I-P X I}{[L W+P X] I}
$$

Y en esta ecuación-conclusión vemos de nuevo algo sorprendente para los usos de neoliberales de la economía y es la de que para que ¡la tasa de ganancia (ahora una sola) $\boldsymbol{g}$ de la economía fuera mayor que cero habría de ocurrir que las inversiones $\boldsymbol{I}_{k}$, más el déficit público $G_{\mathrm{P}}-\mathbf{T}$, más el superávit $\boldsymbol{E}_{\mathrm{X}}$ $\boldsymbol{I}_{\mathrm{M}}$, superaran una parte de las rentas salariales que nos da (1-a) $\boldsymbol{L} \boldsymbol{M}$ más todos los costes de la economía que permiten la reproducción del sistemas $(\boldsymbol{P X I})$ ! Aun cuando queda claro que las tasas de ganancia y de salarios guardan una relación inversa, poco se podía sospechar que para que las empresas tuvieran ganancias se habría de incurrir en un déficit público que supere una parte de las rentas salariales si -por ejemplo- la inversión fuera cero y la balanza comercial estuviera equilibrada. Ello no sería necesario si todas las rentas salariales fueran al consumo, es decir, si a fuera igual a 1. Para llegar a este resultado las matemáticas han sido imprescindibles aún cuando -insisto- a veces no podamos entender los razonamientos económicos que subyacen en las conclusiones formales. En concreto, si todas las rentas salariales se dedicaran al consumo (es decir, si $\mathbf{a}=\mathbf{1}$ ), ocurriría que:

$$
\text { si } a=1 \Rightarrow I_{K}+G_{P}-T+E_{X}-I_{M}>P X I \text { para que } g>0
$$

\footnotetext{
${ }^{16}$ Hay que insistir que, dada la simetría del modelo en lo que atañe a la diferenciación del tipo de ganancias según las condiciones de competencia del mercado expresado por la ecuación (1), todo lo que digamos de un tipo de ganancias lo podemos decir de la otra siempre que ambas estén presentes.
} 
que es una conclusión anti-neoclásica, porque nos dice que si la suma las inversiones más el superávit de balanza de pagos no son suficientes para superar los costes de producción del sistema que permite su reproducción $(\boldsymbol{P X I})$, entonces el Estado ha de incurrir en un déficit presupuestario si se quiere que la economía genere una tasa de ganancia positiva. La razón económica de esta aparente paradoja -lo son todas, claro- es la de que si la economía ha de reproducir el ciclo económico con equilibrio entre oferta y demanda agregadas (estamos en esta hipótesis) un exceso de ganancias lo será a costa de las rentas salariales, rentas que cuanto más altas sean más alto será el consumo y, por tanto, más alta la demanda agregada que pueda permitir igualar a la oferta y reproducir el sistema en las mismas condiciones. Por último también va en la línea antineoclásica el hecho de que cuanto más alto es el coeficiente a o propensión al consumo de las rentas salariales, más alta es la tasa de ganancia, conclusión, en cambio, que se aviene muy bien con los modelos keynesiano/kaleckianos de la demanda efectiva.

Visto (26) desde el lado de las ganancias, es decir, considerada la tasa media de ganancia $\boldsymbol{g}$ como variable dependiente, vamos ahora a darle la vuelta y verlo desde el lado de los salarios. De (26) se despejan los salarios, es decir, la masa salarial $\boldsymbol{L} \boldsymbol{W}$ y queda:

$$
L W I=\left[\frac{1}{(1-a)+(1-b) g}\right] \times\left[I_{K}+G_{P}-T+E_{X}-I_{M}-[1+(1-b) g] P X I\right]
$$

de donde se pueden extraer las siguientes conclusiones: 1) Las masas salariales $\boldsymbol{L} \boldsymbol{M}$ crecen con un aumento de la propensión al consumo de las rentas salariales a, con ¡las inversiones!, jel déficit presupuestario! y el saldo positivo de la balanza comercial; 2) Entre las rentas salariales y las propensiones al consumo hay una asimetría: mientras estas rentas son crecientes en relación a la propensión al consumo de las rentas no salariales, es decreciente respecto a las salariales; 3) Para que las masas salariales sean positivas -lo contrario no tiene sentido económico- ¡el conjunto de las inversiones, más el déficit presupuestario, más el superávit comercial ha de superar el valor agregado de los medios de producción! que permite la reproducción del sistema; formalmente ha de ocurrir que $\left.I_{K}+G_{P}-T+E_{X}-I_{M}>(1+(1-b) g) P X I I ; 4\right)$ Aparece ahora un multiplicador de los salarios que está formado por el primer corchete del lado derecho de la ecuación (28) originado por las propensiones al consumo de las rentas salariales $\boldsymbol{a}$ y por las rentas no salariales $\boldsymbol{b}$, si bien con distinto peso. En efecto, el multiplicador de las rentas no salariales $\boldsymbol{b}$ viene compensado en parte porque aparece en el sustraendo de los sumandos contenidos en el segundo corchete de (28). 


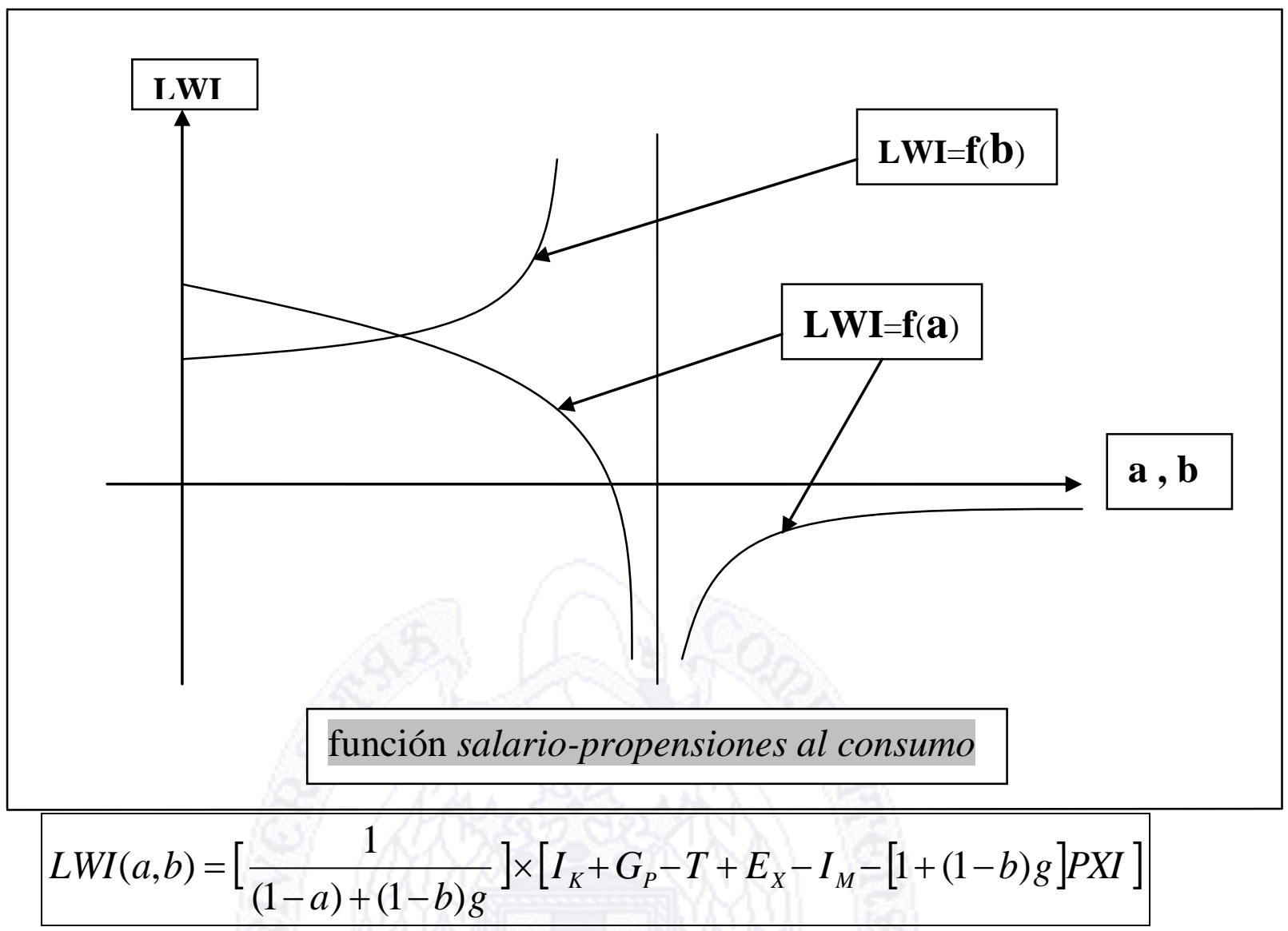

La ecuación (28) merece una reflexión sobre su oportunidad de formar parte de un modelo esrafiano o no esrafiano. La apariencia es que no porque la masa de salarios $L W I$ aparece formalmente en el lado izquierdo de la ecuación como variable (variables) independiente/s. Pero esto es sólo la apariencia porque lo único que indica es que si que quiere una economía definida por las ecuaciones precedentes ya definidas ha de cumplirse que la relación entre las variables $\boldsymbol{a}, \boldsymbol{b}, \boldsymbol{g}, \boldsymbol{I}_{\mathrm{k}}, \boldsymbol{G}_{\mathrm{p}}, \boldsymbol{T}, \boldsymbol{E}_{\mathrm{X}}, \boldsymbol{I}_{\mathrm{M}}, \boldsymbol{P}, \boldsymbol{X}, \boldsymbol{L}, \boldsymbol{W}$, han de satisfacer (28). Las matemáticas no imponen condiciones de causa-efecto sino tan sólo de relación. Es el sentido económico el que pone esa tarea. Incluso (28) nos permite introducir cierta flexibilidad en los precios que permita hacer cumplir la ecuación aún cuando haya que buscar la explicación económica fuera de la ecuación, pero con los límites que esta impone. Si hacemos $\boldsymbol{L} \boldsymbol{W}=\boldsymbol{w} \boldsymbol{L} \mathbf{I}$, es decir, si pasamos de la matriz de salarios a la tasa media ponderada de salarios $\boldsymbol{w}$. Con ello la (28) quedaría:

(28bis)

$\frac{w}{L I}=\hat{w}=\left[\frac{1}{(1-a)+(1-b) g}\right] \times\left[I_{K}+G_{P}-T+E_{X}-I_{M}-[1+(1-b) g] \frac{P X I}{L I}\right]$

Y como quiera que no hemos empleado aún ningún numerario para relativizar las variables, podemos tomar como numerario $P \mathbf{X I} / \mathbf{L I}$, es decir, hacer que $P X \mathbf{L} / \mathbf{L I}=1$. El resultado sería, eso sí, esrafiano, puesto que habríamos eliminado uno de los tótem del italiano: los precios en las variables que definen sus sistema -los sistemas- que pueden deducirse de sus principios, como es el 
caso que nos ocupa. Aún así, lo que importa no es tanto si esrafiano o no esrafiano, sino si contrastable y explicativo o no, si aporta al conocimiento contrastable (científico) o no, y (28) y (28bis) puede ser lanzado a la arena del popperismo para tal menester.

\section{Incorporación furtiva de la inversión}

Lo que se avecina es una sorpresa, pero no adelanto el resultado. Frente a la teoría más keynesiana que relaciona el tipo de interés (causa) con la inversión ${ }^{17}$ (efecto) o la más kaleckiana de relacionar los aumentos del stock de capital (causas) con la inversión ${ }^{18}$ también, voy a seguir un esquema que relaciona el coeficiente capital/trabajo (efecto) con las ganancias (causa), de forma análoga a como también lo hace Kalecki en su teoría dinámica ${ }^{19}$, sólo que con la inversión, como queda dicho. No se pueden quejar los neoclásicos porque parto de la conclusión de Samuelson en su famoso artículo sobre la producción subrogada ${ }^{20}$. Sí, en cambio, debería pedir disculpas a los posibles esrafianos -si es que los hay- por acoplar este paradigma-conclusión neoclásica al presente modelo, y más sabiendo que ya ha sido refutado por Robinson ${ }^{21}$, Sraffa ${ }^{22}$, Pasinetti ${ }^{23}$, Garegnani ${ }^{24}$, Nuti ${ }^{25}$, etc., y que ya forma parte de los intentos fallidos de justificar el paradigma neoclásico-marginalista. Que dicho sea de paso, eso no evita que aún forme parte de los estudios de ortodoxia económica en las facultades de todo el mundo, que es como si en las facultades de Biología se estudiara esta como estaba antes de Darwin, Wallace y la genética evolucionista. Sin más dilación y consideraciones que son más propias de un historiador del análisis económico, introduzco una relación formal -a partir de fundamentos esrafianos- que nos dice que las ganancias (causa) son proporcionales a la relación capital/trabajo (efecto). Al ser proporcionales se huye de la consideración neoclásica de que son crecientemente decrecientes cual si fueran rendimientos (intensivos) de la tierra ricardiana, que es de donde arranca el cáncer marginalista; y sin embargo se acepta que sin ganancias no se recupera en capital (medios de producción), sea cual sea el modelo. Es discutible, desde luego, pero lo es más si puede ser aceptado como esrafiano. Me arriesgo porque en ciencia importa más si es o no oportuno y no la traición. Habría que fechar las variables de la relación pero, dado que estamos en un sistema de equilibrio y de reproducción (ambos no son la misma cosa), no lo hacemos. Esta relación la expresamos en principio de la manera que sigue:

\footnotetext{
17 Teoría general de la ocupación, el interés y el dinero, ed. FCE, pág. 129, 1992.

18 Teoría de la Dinámica Económica, ed. FCE, pág. 99, 1973 \{Theory of Economic Dinamics, $1954\}$.

${ }^{19}$ Obra mencionada, mismas páginas.

20 Parable and Realism in Capital Theory: The surrogate Production Function, 1961.

21 The Production Function and the Theory of Capital, 1953.

22 Production commodities by means commodities, 1960.

${ }^{23}$ Switches of Technique and the "Rate of Return" in Capital Theory, 1969.

${ }^{24}$ Heterogeneous Capital, The Production Function and the Theory Distribuction, 1970.

${ }^{25}$ Capitalism, Socialism and Sleady Growth, 1970.
} 


$$
\frac{P X I}{L I}=M G I
$$

donde $\boldsymbol{P} \boldsymbol{X I}$ es el valor monetario de los medios de producción esrafianos, $\mathbf{L I}$ la suma de los inputs de trabajo, $\boldsymbol{M}$ un vector $\mathbf{1} \times \boldsymbol{n}$ que expresa una relación proporcional positiva con las ganancias, que son, claro está, $G$, que se recordará es una matriz $\boldsymbol{n \times n}$. Por último, recordar también que $\mathbf{I}$ es el vector vertical de unos $(\boldsymbol{n} \times \mathbf{1})$. Si ahora despejamos $\boldsymbol{P X I}$ de (29) y con algunas transformaciones o consideraciones nos queda:

$$
P X I=g L T I
$$

donde $\boldsymbol{T}$ es una matriz $\boldsymbol{n} \times \boldsymbol{n}$ que debe permitir hacer $g \boldsymbol{L} \boldsymbol{T}=M G I L I$. No es necesario calcularlo, sólo saber que es posible encontrarlo ${ }^{26}$ a partir del vector $\boldsymbol{M}$ de (29), de tal forma que se pueda utilizar la misma tasa de ganancia de (28) en (30). Pues bien, si ahora sustituimos (30) en (28) el resultado es como sigue:

$$
L W I=\left[\frac{1}{(1-a)+(1-b) g}\right] \times\left[I_{K}+G_{P}-T+E_{X}-I_{M}-[1+(1-b) g] g L T I\right]
$$

En (31) vemos el cambio extraordinario que se ha producido en las masas salariales, porque éstas ya no dependen linealmente -inversamente sí, clarode la tasa de salarios $\boldsymbol{g}$, sino de forma jcrecientemente decreciente! Más claro se verá si trocamos sin pérdida de generalidad $\boldsymbol{L} \boldsymbol{M}$ por $\boldsymbol{w L I}$, siendo ahora $\boldsymbol{w}$ una (también) sola tasa de salarios. No se produce pérdida de generalidad simplemente porque la tasa $\boldsymbol{w}$ resulta ser una media ponderada de de los salarios de la matriz $\boldsymbol{W}$ de salarios, donde los pesos son los inputs de trabajo $L$ de acuerdo con:

$$
w=\frac{L W I}{L I}
$$

El resultado de la sustitución es:

$$
w=\left[\frac{1}{(1-a)+(1-b) g}\right] \times\left[\frac{I_{K}+G_{P}-T+E_{X}-I_{M}}{L I}-[1+(1-b) g] g \frac{L T I}{L I}\right]
$$

Y sólo queda reiterar lo dicho, porque la relación salario-ganancia en (33) puede ser decrecientemente creciente (en el numerador la tasa de ganancia $\boldsymbol{g}$ está elevada a la segunda potencia si deshacemos el corchete interno), es decir, esta relación es o puede ser ¡cóncava! en lugar de convexa en función

\footnotetext{
${ }^{26}$ En realidad hay $\mathbf{n}-\mathbf{1}$ posibles valores distintos que cumple $\mathbf{g L T I}=\mathbf{M G I L I}$
} 
de los valores de las variables de $(33)^{27}$. Es una refutación -una más- del paradigma de la teoría del capital ja partir de sus propias conclusiones!, que exige un decrecimiento suave entre capital y trabajo en relación a su retribución (tasa de ganancia), es decir, que su segunda derivada sea positiva. De (33) ya hemos obtenido algunas conclusiones antineoclásicas de política económica, pero una de la que no hemos dicho nada es la de que los coeficientes $\boldsymbol{a}$ y $\boldsymbol{b}$ no pueden ser, simultáneamente, igual a $\mathbf{1}$, es decir, que no puede destinarse todas las rentas salariales y no salariales al consumo porque los salarios aumentarían exponencialmente, lo cual no es acorde con la realidad. Ello indica, de alguna manera y en aras del realismo, que nos falta alguna relación fundamental entre las variables que no hemos tenido en cuenta. La razón de ello es que apenas hemos hecho hipótesis restrictivas sobre las variables. Dicho de otra manera, el modelo expresado en (33) es extraordinariamente realista ${ }^{28}$ en el sentido de que se pueden incorporar los datos de la realidad sin apenas hipótesis de comportamiento específicas. Y, sin embargo, a pesar de estas pocas hipótesis (restricciones) de comportamiento o de la generalidad de estas, las conclusiones que se obtienen no son triviales. Es más, diría que son útiles desde la perspectiva del gobierno de la cosa pública al estar implicados el gasto público $G_{p}$ y los impuestos $\boldsymbol{T}$. Parece claro que una de esas ecuaciones de comportamiento omitidas debería referirse al cómo se financia los impuestos.

Y hemos dejado para finalizar el presente epígrafe aún otra sorpresa que no hubiera sido posible intuir sin la ayuda del aparato formal que, aunque elemental, hemos utilizado profusamente. Si llamamos $f$ a $L \mathbf{Y} / \mathbf{L I}$ y despejamos la tasa de ganancia $\boldsymbol{g}$ de (33) obtenemos:

$$
g=\frac{[(1-b)+f] \pm \sqrt{[(1-b)+f]^{2}+4(1-b) f \times\left[\left(I_{K}+G_{P}-T+E_{X}-I_{M}\right) \times \frac{1}{L I}-(1-a) w\right]}}{2(1-b) f}
$$

Lo extraordinario de (34) es que para la tasa de ganancia $\boldsymbol{g}$ sea positiva ha de ocurrir que:

para que $g>0$ ha de ocurrir que $I_{k}+G_{P}-T+E_{X}-I_{M}>(1-a) L W I$

Si se toma la solución en la que la raíz cuadrada de la expresión (34) va afectada con el signo negativo, tiene una lógica económica si no se padece del

\footnotetext{
${ }^{27}$ Dicho en términos matemáticos, la segunda derivada de la tasa del salario respecto a la tasa de ganancia puede ser negativa (cóncava) en lugar de positiva (convexa). Incluso podría pasar de la una a la otra según valores de las variables implicadas en (33). Se deja a la paciencia del lector el cálculo de la primera y segunda derivadas.

${ }^{28}$ Recordar que las tasas de salario y de ganancia no son tasas arbitrarias, sino medias ponderadas de matrices de datos que pueden ser incorporados del mundo real sin apenas reelaboración.
} 
cáncer neoclásico-marginalista. Lo que viene a decir (34) es que, puesto que estamos en un sistema de reproducción de equilibrio, la forma de garantizar las ganancias es que los componentes de la demanda agregada -aunque sea sin el Consumo- sean positivos y que alcancen el mínimo señalado por la ecuación; con ello se estimulará la demanda y la producción. En realidad no nos salimos de lo que ya descubrieron los fisiócratas y de las aportaciones de Keynes, Kalecki y Marx sobre la demanda efectiva y el papel del Consumo. Si esta inversión se redujera a cero, entonces el Estado debería incurrir en un déficit si la balanza comercial no lo remediara con un superávit. Pero los neoclásicos-marginalistas siguen creyendo en la ley de Say, aunque sea sotto voce. Este simple modelo obtenido apenas sin funciones de comportamiento específicas, salvo el de la formación de los precios a partir de un margen sobre todos los costes (pre-factum) y el de la inversión en medios como proporcional a las ganancias obtenidas, nos da una recetas de política económica contrarias a las neoclásicas (neoliberales en lo político) que son contrarias a las que aplican los economistas y las instituciones internacionales (FMI, Reserva Federal, BCE, etc.) para "combatir" la crisis, con los nefastos resultados que están a la vista. Todo lo dicho hasta ahora no son sólo juegos de artificio intelectuales, sino también pedreste recetario de política económica directamente aplicable y verificable.

\section{Dos casos concretos a partir de Sraffa y Pasinetti}

\section{Modelo I.}

En el capítulo VIII del libro de Sraffa Producción de mercancías por medio de mercancías acepta por fin y aunque sea a regañadientes ${ }^{29}$ la definición de bienes no básicos, siendo estos últimos aquellos que nunca entran como medio de producción ni en su sector (o mercancía) ni en los de los demás. Con este criterio económico, Pasinetti se da cuenta en Lecciones de la teoría de la producción $^{30}$ que esta distinción entre bienes básicos y no básicos fijada definitivamente por Sraffa se corresponde con la distinción que hacían los clásicos entre bienes de "primera necesidad" y "bienes de lujo", pero con una novedad que surge del análisis de Sraffa: que ahora la distinción no se hace por las características de los bienes desde el lado del consumo, sino por sus características, por el lado de la producción. A partir de ahí Pasinetti hace un análisis formal de las consecuencias para el sistema de precios y de distribución de la renta. Sin embargo, su efecto es limitado porque el modelo del que parte es el de producción conjunta esrafiano, que es un caso particular y muy restrictivo de todos los posibles. Lo es al menos por dos cosas: a) Sraffa y Pasinetti trabajan con un solo conjunto de ecuaciones de equilibrio, en el que los precios son los mismos cuando entran como medios que cuando entran

\footnotetext{
29 "La distinción entre productos básicos y no básicos se ha hecho tan abstracta en el sistema de productos múltiples () que cabe preguntarse si ha conservado algún contenido económico", pág. 80 en obra citada, edit. Oikos-Tau. En este epígrafe se verá que sí es importante esa distinción al ampliar el estrecho horizonte de la producción conjunta en el que se mueve el extraordinario libro del italiano.

${ }^{30}$ Pág. 139 de la obra citada, edit. FCE, 1983 \{Lezioni di teoria delle Produzione, 1975\}.
} 
como productos; b) las matrices de medios y de productos finales son cuadradas, es decir, hay el mismo número cualitativo de medios de producción que de productos finales. Sin embargo, el camino emprendido resulta productivo porque permite distinguir o distribuir la economía en cuatro sectores según que: a) todos los sectores (mercancías) se compran y se vendan entre sí; b) un conjunto de sectores compran a los demás pero sólo se venden a sí mismos; c) otro conjunto venden a los demás pero sólo se compran a sí mismos; d) un cuarto sector que puede estar separado de los demás en cuanto que no compra ni vende a los demás (que se corresponde con el sector de las "habas" del apéndice B del libro de Sraffa), pero que tiene que aceptar las tasas de salario y ganancia del sector a) que es el único que puede determinar un conjunto de precios, salarios y ganancias de equilibrio (es decir, de reproducción del sistema) sin depender de los demás. Veamos ahora cómo podría formalizarse el caso b) de Pasinetti o que podría deducirse de sus meras consideraciones matemáticas pero ampliando el horizonte. Este modelo $^{31}$ que podríamos tildar de Sraffa-Pasinetti vendría definido por la siguientes ecuaciones:

$$
P_{A(1 x u)}\left(Y_{A(u x n)}+Y_{B(u x n)}\right)=\left[L_{A(1 x u)} W_{A(u x n)}+P_{A(1 x u)} X_{A(u x n)}\right]\left(I_{d(n x n)}+G_{A(n x n)}\right)
$$

$$
P_{Z(1 x(m-u))} Z_{(m-u) x n}=\left[L_{B(1 x(m-u))} W_{B((m-u) x n)}+P_{A(1 x u)} Y_{B(u x n)}\right]\left(I_{d(n x n)}+G_{B(n x n)}\right)
$$

donde los precios $\boldsymbol{P}_{\mathrm{A}}$ y los inputs de trabajo $\boldsymbol{L}_{\mathrm{A}}$ están definidos por un vector $\mathbf{1} \boldsymbol{u}$; los productos finales $\boldsymbol{Y}_{\mathrm{A}}$, los medios de producción $\boldsymbol{X}_{\mathrm{A}}, \boldsymbol{Y}_{\mathrm{B}}$ y los salarios $\boldsymbol{W}_{\mathrm{A}}$ son matrices $\boldsymbol{U} \times \boldsymbol{n}$, y las tasas de ganancia $\boldsymbol{G}_{A}$ lo están por una matriz $\boldsymbol{n} \times \boldsymbol{n}$; por otro lado, los precios $\boldsymbol{P}_{\mathrm{B}}$ y los inputs de trabajo $L_{\mathrm{B}}$ lo están por un vector $\mathbf{1} \times(\boldsymbol{m}$ $\boldsymbol{u}$ ); los productos finales $\boldsymbol{Z}$, los medios de producción (pero productos finales en la ecuación (36)) $\boldsymbol{Y}_{\mathrm{B}}$ y los salarios $\boldsymbol{W}_{\mathrm{B}}$ son matrices $(\boldsymbol{m}-\boldsymbol{u}) \times \boldsymbol{n}$, y donde, para acabar, las ganancias $\boldsymbol{G}_{B}$ se definen también por una matriz $\boldsymbol{n} \times \boldsymbol{n}$. Es decir, el conjunto de sectores (mercancías o bienes y servicios) definidos por la ecuación (36) produce los productos $\boldsymbol{Y}_{\mathrm{A}}$ que van a entrar como medios de producción en el período siguiente (estamos con precios y cantidades de equilibrio), pero también producen los bienes y servicios $\boldsymbol{Y}_{\mathrm{B}}$ que van a servir como medios de producción en el sector no-básico definido por la ecuación (37). Los bienes y servicios de la (36) lo serían de bienes básicos. Puede comprobarse en el conjunto de ecuaciones (36) y (37) el avance sobre la modelización de Pasinetti a partir de Sraffa: 1) la producción de bienes básicos $\left(\begin{array}{lll}\boldsymbol{Y}_{\mathrm{A}} & \text { y } & \boldsymbol{Y}_{\mathrm{B}}\end{array}\right)$ se distingue implícitamente de la de bienes no-básicos $\boldsymbol{Z}$ no sólo cualitativamente, sino formalmente al estar separados en dos ecuaciones; 2) las matrices de productos y medios que definen el sistema no son cuadradas, lo cual es un avance extraordinario en pos del realismo de las hipótesis. En (36) ya se puede comprobar a simple vista que, bajo ciertas condiciones, los ${ }^{31}$ Ellos no han desarrollado nada de lo que viene, por lo que no se les puede hacer
responsables y menos culpables de lo que viene. 
precios pueden determinarse autónomamente sin necesidad de recurrir a (37). No ocurre lo mismo con (37), es decir, en las ecuaciones que definen los sectores (mercancías) no-básicas, porque los productos finales $\boldsymbol{Z}$ son función (dependen) de los precios $\boldsymbol{P}_{\mathrm{A}}$ y de los productos finales $\boldsymbol{Y}_{\mathrm{B}}$ de la ecuación (36), pero que son precios y medios en la (37). Aunque luego pasaremos a tasas únicas (medias) de salarios y ganancias, vamos a formalizar esta cuestión para que se entienda todo esto. Si hacemos cero todas las tasas de ganancia $\boldsymbol{W}_{\mathrm{A}}$ pasar obtener las tasas de ganancia máximas ${ }^{32} \boldsymbol{B}$ tenemos la ecuación:

$$
P_{A}\left(Y_{A}+Y_{B}\right)=P_{A} X_{A}\left(I+B_{A}\right)
$$

Pues bien, ahora de (36) y (37) surge la ecuación de determinación de los precios:

$$
P_{A}=L_{A} W_{A}\left(I+G_{A}\right)\left(B_{A}-G_{A}\right)^{-1} X_{A}^{T}\left(X_{A} X_{A}^{T}\right)^{-1}
$$

que sustituida en (37) obtenemos los precios $\boldsymbol{P}_{\mathbf{Z}}$ del sector de bienes nobasicos dependiente de salarios, medios y ganancias del sector de básicos (36), además de las tasas de salarios, medios y ganancias propias, sea cuales sean los precios del sector de bienes básicos. Pero no es el objetivo de este trabajo ni de este epígrafe una posible determinación de los precios en este modelo de producción conjunta no esrafiana a partir de la distinción entre bienes básicos y no-básicos. A pesar del trabajo y del acento puesto por el gran historiados del análisis económico Alexandro Roncaglia, yo no creo que lo sustantivo -ni en la intención ni en los resultados- en la obra de Sraffa sea el tema de los precios su principal preocupación. Creo que esta perspectiva está marcada -quizá inconscientemente- en la necesidad de presentar una alternativa a los modelos de equilibrio general de origen walrasiano o posteriores (Debreu, Arrow). El núcleo duro y la preocupación de la obra de Sraffa en Producción de mercancías por medio de mercancías es el excedente, su reparto y sus límites. Por eso y siguiendo la introducción de este artículo, vamos a dar un giro al tema y centrarnos en las consecuencias del reparto del excedente a partir de este modelo de economía formalizado en dos ecuaciones matriciales, una de bienes básicos y otra de bienes no-básicos. Para ello vamos a trabajar con tasas de ganancia y de salario únicas, pero representativas de toda la economía, a diferencia de cómo lo hace el propio Sraffa, Pasinetti, Schefold, Kurz, etc. En efecto, de forma análoga ha como lo hemos hecho en las ecuaciones (22) y (23) vamos a pasar a unas tasas de ganancia $\boldsymbol{g}_{\mathrm{A}}$ y $\boldsymbol{w}_{\mathrm{A}}$ unitarias tales que cumplan con las ecuaciones:

$$
\begin{aligned}
& L_{A} W_{A}\left(I+G_{A}\right) I=w_{A}\left(1+g_{A}\right) L_{A} I \\
& P_{A} X_{A}\left(I+G_{A}\right) I=\left(1+g_{A}\right) P_{A} X_{A} I
\end{aligned}
$$

\footnotetext{
${ }^{32}$ Las tasas máximas de ganancia en la producción conjunto esrafiana y no esrafiana son el equivalente a la razón-patrón en la producción simple de Sraffa, donde se recordará que la matriz de bienes finales es una matriz diagonal con ceros en los elementos donde la fila es distinta de la columna y donde la matriz de medios es cuadrada.
} 
donde I es el vector vertical $n \times 1$ de unos, con lo que (40) y (41) son escalares, razón de lo cual $\boldsymbol{w}_{\mathrm{A}}$ y $\boldsymbol{g}_{\mathrm{A}}$ son las tasas medias y únicas mencionadas y con $\boldsymbol{g}_{\mathrm{mA}}$ como tasa unitaria máxima del sector de bienes básicos ${ }^{33}$. Y ahora, entre (37), (39), (40) y (41) obtenemos como paso intermedio:

$$
P_{Z} Z I=\left(1+g_{B}\right) w_{B} L_{B} I+\frac{w_{A}\left(1+g_{A}\right)\left(1+g_{B}\right)}{g_{m A}-g_{A}} \times L_{A} X_{A}^{T}\left(X_{A} X_{A}^{T}\right)^{-1} Y_{A} I
$$

Y si ahora dividimos (42) entre $\boldsymbol{P}_{\mathbf{Z}} \mathbf{Z} \mathbf{I}$ y para facilitar la expresión de las ecuaciones ${ }^{34}$ hacemos:

$$
\begin{aligned}
& F_{1}=\frac{L_{B} I}{P_{Z} Z I} \\
& F_{2}=\frac{L_{A} X_{A}^{T}\left(X_{A} X_{A}^{T}\right)^{-1} Y_{A} I}{P_{Z} Z I}
\end{aligned}
$$

y despejamos la tasa de ganancia del sector de bienes no-básicos $g_{\mathrm{B}}$

$$
g_{B}=\frac{\left(1-w_{B} F_{1}\right) g_{m A}-w_{A} F_{2}-\left[1-w_{B} F_{1}+w_{A} F_{2}\right] g_{A}}{w_{B} g_{m A} F_{1}+w_{A} F_{2}+\left[w_{A} F_{2}-w_{B} F_{1}\right] g_{A}}
$$

En (45) se comprueba que la tasa de ganancia -así como antes en (42) los precios $\boldsymbol{P}_{\boldsymbol{Z}}$ - depende de la tasa de ganancia $\boldsymbol{g}_{\mathrm{A}}$ obtenida en el sector de bienes básicos. Podemos decir que este sector es ganancia-aceptante, de forma análoga a como lo son los precios en los modelos de competencia perfecta marginalista, donde el óptimo del nivel de producción se obtiene al igualar estos precios con los costes marginales. Veamos algunas de sus características: 1) Si se hallan las primera y segunda derivadas de la tasa de ganancia $g_{\mathrm{B}}$ (bienes no-básicos) respecto a la otra tasa $\boldsymbol{g}_{\mathrm{A}}$ (básicos) se vera que la primera es negativa y la segunda positiva, por lo que la función que representa (45) es convexa, lo que significa que un aumento de la tasa de ganancia de los productos básicos $\boldsymbol{g}_{\mathrm{A}}$ provoca una caída menos pronunciada en la tasa de ganancia de los no-básicos; 2) Los puntos de corte de la función (45) son:

$$
\begin{gathered}
g_{B}\left(g_{A}=0\right)=\frac{\left(1-w_{B} F_{1}\right) g_{m A}-w_{A} F_{2}}{w_{B} g_{m A} F_{1}+w_{A} F_{2}} \\
g_{A}\left(g_{B}=0\right)=\frac{\left(1-w_{B} F_{1}\right) g_{m A}-w_{A} F_{2}}{1-w_{B} g_{m A} F_{1}+w_{A} F_{2}}
\end{gathered}
$$

\footnotetext{
${ }^{33}$ Esta tasa no se deduce de ninguna matriz de tasas medias y hay que suponerla ad hoc.

34 Tanbién podemos tomar como numerario $\boldsymbol{P}_{\mathbf{Z}} \mathbf{Z I}$, es decir, hacer $\boldsymbol{P}_{\mathbf{Z}} \mathbf{Z I}=\mathbf{1}$, con lo cual eliminamos el único vector de precios que aún tenemos.
} 
lo que significa que para ambas tasas de ganancia sean positivas ha de ocurrir que la tasa de salarios del sector de bienes no-básicos $\boldsymbol{w}_{\mathrm{B}}$ no puede superar ni igualar el límite marcado por $\left(1-\boldsymbol{w}_{\mathrm{A}} \boldsymbol{F}_{2} / \boldsymbol{g}_{\mathrm{mA}}\right)$ y que la tasa de salarios de los sectores básicos no supere o iguale el cociente $\boldsymbol{g}_{\mathrm{mA}} / \boldsymbol{F}_{2}$; 3 ) En general, para que la tasa de ganancia de los básicos $g_{\mathrm{B}}$ sea positiva ha de ocurrir según (45) que:

$$
g_{A}<\frac{\left(1-w_{B} F_{1}\right) g_{m A}-w_{A} F_{2}}{1-w_{B} g_{m A} F_{1}+w_{A} F_{2}}
$$

lo que implica a su vez que la tasa de salario medio (único) del sector nobásico $\boldsymbol{w}_{\mathrm{B}}$ permanezca por debajo de:

$$
w_{B}<\frac{1}{F_{1}}-\frac{w_{A} F_{2}}{g_{m A} F_{1}}
$$

y que para que esta tasa de salarios sea positiva ha de ocurrir que los salarios de los no-básicos $\boldsymbol{w}_{\mathrm{A}}$ quede por debajo de:

$$
w_{A}<\frac{g_{m A}}{F_{2}}
$$

La conclusión es que la tasa de ganancia del sector de bienes no-básicos $\boldsymbol{g}_{\mathrm{B}}$ depende enteramente de la de los básicos $\boldsymbol{g}_{\mathrm{A}}$, que esta se determina autónomamente con (36) y (38) y que sus márgenes pueden ser muy estrechos en función de las variables que están en (45), que son, del sector de básicos, la tasa de salarios $\boldsymbol{w}_{\mathrm{A}}$, el tipo de ganancia máxima $\boldsymbol{g}_{\mathrm{mA}}$, los inputs de trabajo $\boldsymbol{L}_{\mathrm{A}}$, los medios de producción $\boldsymbol{X}_{\mathrm{A}}$ y los bienes finales $\boldsymbol{Y}_{\mathrm{A}}$, y de la tasa de salarios $\boldsymbol{w}_{\mathrm{B}}$ e inputs de trabajo $L_{B}$ de su propio sector (no-básicos), sean cuales sean los precios de ambos sectores y sea cual sea la producción final del sector de nobásicos $\boldsymbol{Z}$. Hay que recordar que estamos en una situación de equilibrio en precios y de reproducción del sistema en el sector de bienes básicos y ello condiciona la concreción de estas soluciones, pero no las variables que intervienen ni la relación entre ambas tasas de ganancia. 


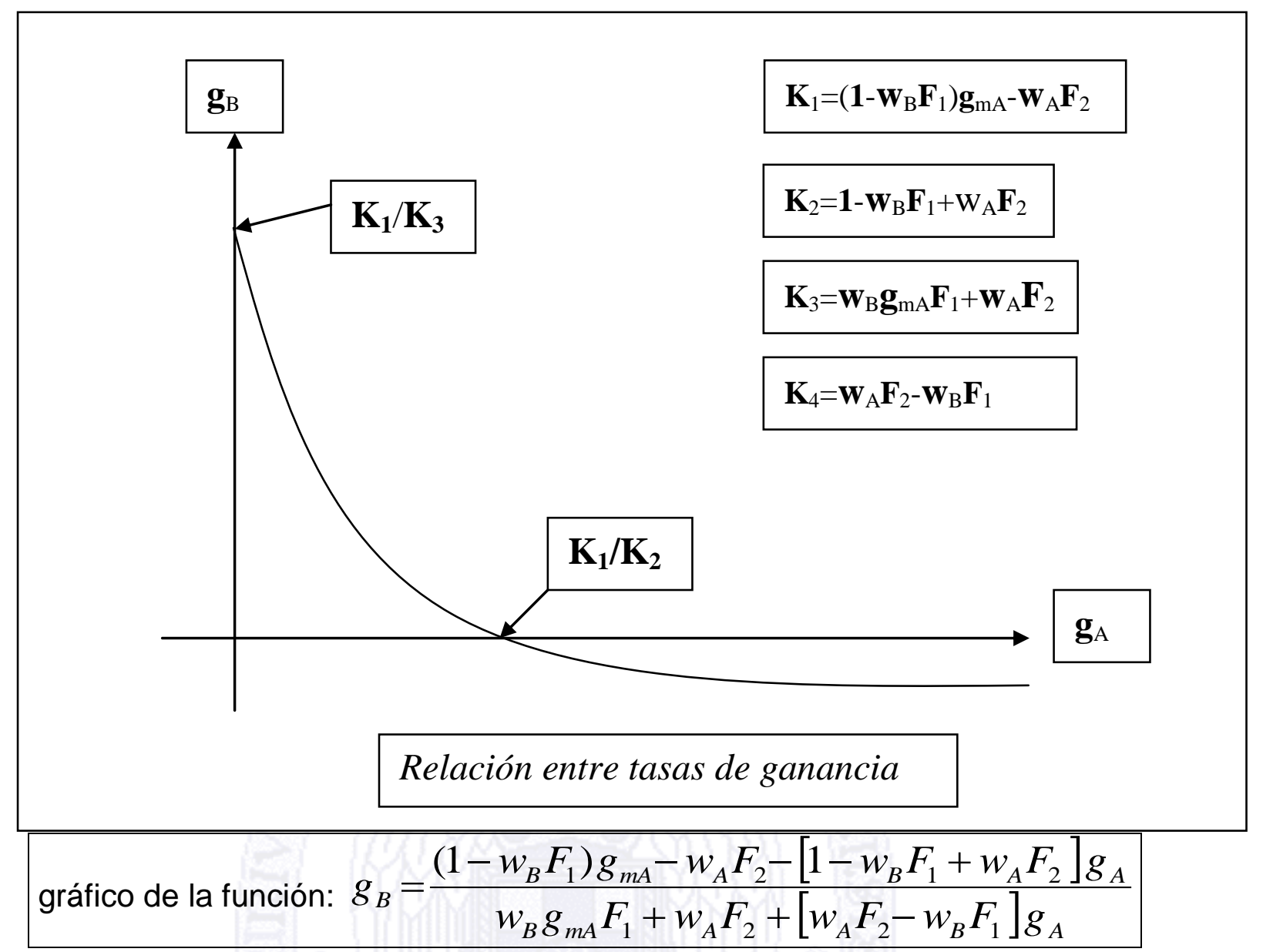

Resulta curioso que, a diferencia de esa relación convexa entre las tasas de ganancia, no ocurra lo mismo con la relación entre los salarios. En efecto, si despejamos de (45) los salarios de los sectores básicos $\boldsymbol{w}_{\mathrm{B}}$ se obtiene:

$$
w_{B}=\frac{1}{\left(1+g_{B}\right) F_{1}}-\frac{1+g_{A}}{g_{m A}-g_{A}} \times \frac{F_{2}}{F_{1}} \times w_{A}
$$

donde puede comprobarse la relación inversa y lineal entre ambas tasas de salario.

En todo lo anterior hemos supuesto que existe la inversa de $\boldsymbol{X}_{\mathrm{A}} \boldsymbol{X}_{\mathrm{A}}{ }^{\top}$, es decir, el producto matricial de la matriz de medios por su traspuesta. En primer lugar, la matriz $\boldsymbol{X}_{\mathrm{A}}$ es una matriz no cuadrada $\boldsymbol{u} \times \boldsymbol{n}$, por lo que sólo podemos asegurar que existe esta inversa si $\boldsymbol{u}$ es menor que $\boldsymbol{n}$, es decir, si el número de bienes y servicios básicos es menor que el número total de sectores de la economía. Con ello aseguramos que el rango de la matriz $\boldsymbol{X}_{\mathrm{A}} \boldsymbol{X}_{\mathrm{A}}{ }^{\top}$ es igual o menor que el menor de los valores $\boldsymbol{u}$ y $\boldsymbol{n}$. Pero esto es problemático y de por sí es un supuesto restrictivo. En segundo lugar, nada nos asegura que todos los elementos surgidos del cálculo de la inversa de $\boldsymbol{X}_{\mathrm{A}} \boldsymbol{X}_{\mathrm{A}}{ }^{\top}$ vayan a ser positivos ${ }^{35}$.

\footnotetext{
${ }^{35}$ Al no ser $\mathbf{X}_{\mathrm{A}}$ cuadrada, de entrada, ya no se puede aplicar Rouché-Frobenius, aun cuando la economía la supongamos productiva, es decir, aun cuando se produzca no menos productos en todos los sectores de los que se emplea en el sector correspondiente. Hay que recordar que
} 
Con ello podría ocurrir que algunos de los precios de los productos de bienes básicos $\boldsymbol{P}_{\mathrm{A}} \mathrm{y}$, por lo tanto, de bienes no-básicos fuera negativo. Pero hay que recordar que en este epígrafe -a diferencia de los anteriores- estamos trabajando a nivel agregado, por lo que no cabe pensar que la suma en términos de valor de todos los bienes tales como $\boldsymbol{P}_{\mathrm{A}}\left(\boldsymbol{Y}_{\mathrm{A}}+\boldsymbol{Y}_{\mathrm{B}}\right) \mathbf{I}$ vaya a ser negativa (es un escalar); el mismo caso para $\boldsymbol{P}_{\mathbf{Z}} \boldsymbol{Z}$. Sraffa recurría en estas situaciones a una especie de criterio sociológico empresarial que haría que se desecharan los métodos de producción -representados aquí por las variables $L_{\mathrm{A}}, L_{\mathrm{B}}, \boldsymbol{Y}_{\mathrm{A}}, \boldsymbol{Y}_{\mathrm{B}}, \boldsymbol{Z}$ - que dieran precios negativos, puesto que eso no tiene sentido económico. Podemos recurrir en general a ello, pero no hay que olvidar también que algunos subsectores de la agricultura, productos lácteos o mineros están subvencionados en la economía real para evitar que los costes superen los ingresos.

\section{Modelo II.}

Una generalización del caso anterior sería aquel en el que dividiríamos la economía en dos sectores, uno de competencia según grados y otro abiertamente monopolístico, donde el criterio económico que nos guiaría sería el anunciado en el primer epígrafe de este artículo: el competitivo sería aquel en el que la tasa de ganancia tendiera a cero -al igual que en el esquema marginalista-y el monopolista sería el que pudiera llevar la tasa de ganancia hasta la tasa máxima. Este caso no es equivalente al marginalista, donde el monopolista se enfrenta a una curva de demanda y puede elegir el nivel de producción tal que los ingresos marginales derivado de su producto iguale a los ingresos marginales. Aquí nos olvidamos de la diferenciación entre bienes básicos y no básicos siguiendo el consejo de Schefold y sólo dividimos la economía en dos sectores en función del grado de competencia. Lo nuevo de este modelo respecto a los anteriores es que una parte de lo que produce cada sector forma parte de los medios de producción del otro. Se correspondería a una economía Sraffa-Pasinetti en la que ambos son clientes y proveedores entre sí y también respecto al otro sector. Aquí, por tanto, la simetría es total y sólo son autosuficientes en los productos que sólo se venden a sí mismos pero no respecto al que venden al otro. Ello permite que cada sector tenga su propia matriz de salarios y de ganancias; además permite que lo que producen para sí tengan rangos diferentes; por último permite que las matrices de medios y productos no sean cuadradas, es decir, que el número de sectores y de bienes y servicios producidos (mercancías) no sean iguales. Todo esto supone un gran avance respecto a los modelos de origen esrafiano, incluso los referidos a la producción conjunta; y mayor realismo es ya casi imposible. Sin más dilación exponemos a continuación las ecuaciones que definen el modelo:

$$
P_{C} Y_{C}+P_{A} Y_{A}+P_{B} X_{B}=\left[L_{A} W_{A}+P_{A} X_{A}\right]\left(I+G_{A}\right)
$$

las condiciones necesarias y suficientes para que en una economía esrafiana, es decir, una economía de producción simple, de un vector de precios positivo es que la matriz de requerimientos $\mathbf{A}=\mathbf{X} \mathbf{Y}^{-1}$ sea cuadrada, no negativa, indescomponible. Nada de esto tenemos ahora. 


$$
P_{D} Y_{D}+P_{B} Y_{B}+P_{A} X_{A}=\left[L_{B} W_{B}+P_{B} X_{B}\right]\left(I+G_{B}\right)
$$

donde $\boldsymbol{P}_{\mathrm{A}}, \boldsymbol{L}_{\mathrm{A}}$ son vectores $\mathbf{1} \boldsymbol{x} \boldsymbol{u}, \boldsymbol{Y}_{\mathrm{A}}, \boldsymbol{X}_{\mathrm{A}}, \boldsymbol{W}_{\mathrm{A}}$ son matrices $\boldsymbol{u} \times \boldsymbol{n}, \boldsymbol{P}_{\mathrm{B}}, \boldsymbol{L}_{\mathrm{B}}$ vectores $\mathbf{1} \times(\boldsymbol{m}-\boldsymbol{u}), \boldsymbol{Y}_{\mathrm{B}}, \boldsymbol{X}_{\mathrm{B}}, \boldsymbol{W}_{\mathrm{B}}$ matrices $(\boldsymbol{m}-\boldsymbol{u}) \times \boldsymbol{n}$, y, para acabar, las matrices $\boldsymbol{G}_{\mathrm{A}}$ y $\boldsymbol{G}_{\mathrm{B}}$ tienen dimensión $\boldsymbol{n} \times \boldsymbol{n}$, aunque sean distintas (que pueden suponerse iguales en un momento determinado). Como puede comprobarse en la pareja de ecuaciones matriciales anterior, el valor de medios de producción del sector $\mathbf{A}$ (competitivo) $\boldsymbol{P}_{\mathrm{A}} \boldsymbol{X}_{\mathrm{A}}$ es producido en el sector $\mathbf{B}$ (no competitivo) y el valor de los medios del sector $\mathbf{B}$ que es $\boldsymbol{P}_{\mathrm{B}} \boldsymbol{X}_{\mathrm{B}}$ es producido en el sector $\mathbf{A}$. Los precios $\boldsymbol{P}_{\mathrm{C}}$ y $\boldsymbol{P}_{\mathrm{D}}$ así como sus productos finales $\boldsymbol{Y}_{\mathrm{C}}$ y $\boldsymbol{Y}_{\mathrm{D}}$ son bienes de consumo que producen ambos sectores de la economía, pero que no van a influir en lo que sigue. La razón de ello es que no intervienen como medios sus bienes físicos y tampoco sus precios, tal y como puede comprobarse en (52) y (53). También pueden tener cualquier dimensión entre el vector de precios y su correspondiente matriz de productos con tal de que la dimensión del vector resultante de su producto sea $1 \times n$. Por supuesto que también producen sus propios medios de producción y, por ello, ha de suponerse que $\boldsymbol{Y}_{\mathrm{A}}>\boldsymbol{X}_{\mathrm{A}}$ y que $\boldsymbol{Y}_{\mathrm{B}}>\boldsymbol{X}_{\mathrm{B}}$, aunque no sea estrictamente necesario este supuesto para lo que sigue. Vamos a ver cuál es el resultado analítico de estos supuestos. Si sumamos miembro a miembro las ecuaciones (52) y (53) y eliminamos términos comunes queda (54):

$$
P_{C} Y_{C}+P_{D} Y_{D}+P_{A} Y_{A}+P_{B} Y_{B}=L_{A} W_{A}\left(I+G_{A}\right)+L_{B} W_{B}\left(I+G_{B}\right)+P_{A} X_{A} G_{A}+P_{B} X_{B} G_{B}
$$

En (54) vemos la relación inversa entre ambos tipos de ganancia, entre ambos tipos de salarios y entre ganancias y salarios, pero no podríamos decir mucho más si no pasáramos a trabajar con tasas máximas de ganancia, lo cual nos va a permitir eliminar los precios. También trabajaremos con tasas de salarios y ganancias unitarias, pero, al igual que antes, no tasas unitarias cualesquiera, sino aquellas que se derivan de los datos del problema, es decir, tasas medias ponderadas, tasas por tanto representativas y no meramente arbitrarias. Esto supone también un avance respecto a los modelos con los que trabajan los esrafianos (Schefold, Kurz, Roncaglia, Pasinetti, etc.), que utilizan tasas unitarias ad hoc, lo cual los aleja de la realidad, de la toma de datos de la realidad. Ya hemos hecho con anterioridad estos supuestos pero sin reflexionar sobre sus circunstancias.

$$
\begin{aligned}
& L_{A} W_{A}\left(I+G_{A}\right) I=w_{A}\left(1+g_{A}\right) L_{A} I \\
& L_{B} W_{B}\left(I+G_{B}\right) I=w_{B}\left(1+g_{B}\right) L_{B} I \\
& L_{A} W_{A} G_{A} I=w_{A} g_{A} L_{A} I \\
& L_{B} W_{B} G_{B} I=w_{B} g_{B} L_{B} I
\end{aligned}
$$

Donde I es el vector vertical de unos $n \times 1$, que sirve para sumar los valores de los vectores y matrices de inputs de trabajo, salarios y ganancias y convertirlos en un escalar. Es decir, las 4 ecuaciones anteriores ya no son un sistema de 
ecuaciones matriciales (vectoriales) sino 4 ecuaciones algebraicas de donde se pueden obtener las 4 tasas unitarias de salarios y ganancias $\boldsymbol{w}_{\mathrm{A}}, \boldsymbol{w}_{\mathrm{B}}, \boldsymbol{g}_{\mathrm{A}}, \boldsymbol{g}_{\mathrm{B}}$. $\mathrm{Si}$ ahora tomamos como numerario ${ }^{36}$ en (54) la ecuación queda:

$$
P_{C} Y_{C} I+P_{D} Y_{D} I+P_{A} Y_{A} I+P_{B} Y_{B} I=1
$$

siendo I el vector de unos $\boldsymbol{n \times 1}$. Por último faltan las ecuaciones de precios en función de las tasas matrices $n \times n$ de ganancia máximas $\boldsymbol{G}_{\mathrm{mA}}$ y $\boldsymbol{G}_{\mathrm{mB}}$ que surgen al hacer cero las matrices de salarios en (52) y (53), tal y como hace Sraffa en su obra Producción de mercancías por medio de mercancías. Con ello obtenemos:

$$
\begin{aligned}
& P_{A}=L_{A} W_{A}\left(I+G_{A}\right)\left(G_{m A}-G_{A}\right)^{-1} X_{A}^{-1} \\
& P_{B}=L_{B} W_{B}\left(I+G_{B}\right)\left(G_{m B}-G_{B}\right)^{-1} X_{B}^{-1}
\end{aligned}
$$

Al igual que con las tasas de salario y de ganancias anteriores, ahora podemos pasar de estas matrices de tasas máximas a tasas máximas unitarias mediante las ecuaciones:

$$
\begin{aligned}
& L_{A} W_{A}\left(I+G_{A}\right)\left(G_{m A}-G_{A}\right)^{-1} I=\frac{w_{A}\left(1+g_{A}\right)}{g_{m A}-g_{A}} \times L_{A} I \\
& L_{B} W_{B}\left(I+G_{B}\right)\left(G_{m B}-G_{B}\right)^{-1} I=\frac{w_{B}\left(1+g_{B}\right)}{g_{m B}-g_{B}} \times L_{B} I
\end{aligned}
$$

Pues bien, todas las ecuaciones que van de la (55) a la (63) sustituidas en la (54) pos-multiplicada por el vector I de valores unitarios $n \times 1$ dan como resultado:

$$
g_{B}=\frac{\left[\left[1-w_{A} L_{A} I-w_{B} L_{B} I\right] g_{m A}-\left[1+w_{A} g_{m A} L_{A} I-w_{B} L_{B} I\right] g_{A}\right] g_{m B}}{\left[1-w_{A} L_{A} I+w_{B} g_{m B} L_{B} I\right] g_{m A}-\left[1+w_{A} g_{m A} L_{A} I+w_{B} g_{m B} L_{B} I\right] g_{A}}
$$

que en términos de masas salariales de acuerdo con (57) y (58) sería:

\footnotetext{
${ }^{36}$ Recordar que tomar un numerario en una ecuación - de lo contrario no tiene sentido- supone dividir todos los términos (sumandos) de la ecuación entre la expresión que se toma como tal. Es curioso que esto no suele ser advertido en los artículos y libros, pero ello supone que al menos una parte de las variables ya no son las originales, sino las originales divididas entre el numerario, aunque se sigan utilizando las mismas expresiones gráficas -letras- para estas variables transformadas. Esta advertencia quizá no tenga mucho valor cuando se trabaja con modelos abstractos, pero no en nuestro caso, porque aquí podemos arrancar con valores concretos tomados de la realidad, aunque luego podamos elevarnos -como dirían Marx y Hegel- de lo abstracto a lo concreto.
} 
(64b)

$g_{B}=\frac{\left[\left[1-L_{A} W_{A} I-L_{B} W_{B} I\right] g_{m A}-\left[1+g_{m A} L_{A} W_{A} I-L_{B} W_{B} I\right] g_{A}\right] g_{m B}}{\left[1-L_{A} W_{A} I+g_{m B} L_{B} W_{B} I\right] g_{m A}-\left[1+g_{m A} L_{A} W_{A} I+g_{m B} L_{B} W_{B} I\right] g_{A}}$

La ecuación (64) expresa la dependencia de la tasa de ganancia $g_{\mathrm{B}}$ de los sectores no competitivos de la de los sectores competitivos $\boldsymbol{g}_{\mathrm{A}}$. De (64) podemos obtener las siguientes conclusiones no triviales: 1) Puede comprobarse ique han desaparecido los precios!, cumpliéndose el sueño de Ricardo de encontrar una medida de la distribución independiente de ellos, pero con el añadido de que están implicadas en ese reparto dos tasas de ganancia ${ }^{37}$ y dos tasas de salario. La ecuación tiene un punto de corte tal como:

$$
g_{B}\left(g_{A}=0\right)=\frac{\left[1-w_{A} L_{A} I-w_{B} L_{B} I\right] g_{m B}}{\left[1-w_{A} L_{A} I+w_{B} g_{m B} L_{B} I\right]}
$$

que determina la tasa de ganancia del sector no competitivo $\boldsymbol{g}_{\mathrm{B}}$ cuando la tasa de ganancia del competitivo $\boldsymbol{g}_{\mathrm{A}}$ se hace cero, es decir, cuando se cumple el supuesto de competitividad que hemos recogido desde el principio. El otro punto de corte viene dado por la ecuación:

$$
g_{A}\left(g_{B}=0\right)=\frac{\left[1-w_{A} L_{A} I-w_{B} L_{B} I\right] g_{m A}}{\left[1+w_{A} g_{m A} L_{A} I-w_{B} L_{B} I\right]}
$$

2) Tiene también la función (64) una asíntota de acuerdo con:

$$
\text { limite de } g_{B}\left(g_{A} \rightarrow \infty\right)=\frac{\left[1+w_{A} L_{A} I-w_{B} L_{B} I\right] g_{m B}}{\left[1+w_{A} L_{A} I+w_{B} g_{m B} L_{B} I\right]}
$$

3) Para que el sector no competitivo B obtenga beneficios ha de ocurrir que se cumpla:

$$
g_{B}>0 \text { si } g_{A}<\frac{\left[1-w_{A} L_{A} I-w_{B} L_{B} I\right] g_{m A}}{\left[1+w_{A} g_{m A} L_{A} I-w_{B} L_{B} I\right]}
$$

que indica que ambas tasas de ganancias serán positivas en función de los valores concretos de las tasas de salarios de ambos sectores. En concreto, ambas tasas de ganancias serán positivas si los salarios del sector no competitivo $\boldsymbol{w}_{\mathrm{B}}$ quedan por debajo de las expresiones:

\footnotetext{
${ }^{37}$ No incluyo las tasas de ganancia máximas $\boldsymbol{g}_{\mathrm{mB}}$ y $\boldsymbol{g}_{\mathrm{mA}}$ porque estas, a pesar de su apariencia, no son variables monetarias puesto que su valor depende enteramente y únicamente de los medios de producción y de los productos finales (ver apéndice).
} 


$$
w_{B}<\frac{1-w_{A} L_{A} I}{L_{B} I} \quad \text { y } \quad w_{B}<\frac{1+w_{A} g_{m A} L_{A} I}{L_{B} I}
$$

Si en la ecuación (65) igualamos las dos tasas de salario y despejamos esta variable en función del resto obtenemos:

$$
w\left(g_{A}=0\right)=\frac{g_{m B}-g_{B}}{\left[L_{A} I+L_{B} I\right] g_{m B}+\left[g_{m B} L_{B} I-L_{A} I\right] g_{B}}
$$

Tanto la ecuación (65) como la (70) pueden ser entendidas también como el de la frontera salarios-ganancia. $Y$ aquí tampoco está garantizado que la función sea convexa porque eso sólo ocurre si se cumple que:

$$
g_{m B}>\frac{L_{A} I}{L_{B} I}
$$

Y si es menor (71), es decir, si $\boldsymbol{g}_{\mathrm{mB}}<\boldsymbol{L}_{\mathrm{A}} \mathrm{l} / \boldsymbol{L}_{\mathrm{B}} \mathbf{I}$, ¡ni siquiera podemos asegurar que la función (70) sea decreciente! Un caso particular es el de que si (71) se cumpliera con el signo de igualdad la función frontera salario-ganancia (70) sería una recta (eso sí, decreciente, como cabía esperar). En mi opinión se trata de una de las mejores refutaciones -una más- de la teoría del capital neoclásica por tres razones: 1) porque hemos partido de dos ecuaciones (52) y (53) de definición del sistema tan generalizadas que casi podemos tocar la realidad con los dedos; 2) porque las tasas unitarias medias de ganancias y salarios no suponen ninguna pérdida de generalidad; 3) porque no hay ninguna razón económica para que (71) pueda darse con el signo mayor de desigualdad, aún cuando pueda ser el caso más general.

Un resultado interesante y no evidente cuando comenzábamos este epígrafe es que si en (70) el sector monopolístico pudiera aumentar las ganancias hasta llegar a la tasa máxima ocurriría que no sólo se verían afectados los salarios de este sector, sino que también se verían reducidos a ceros los del sector competitivo (en este esquema particular se han supuesto iguales). La razón económica es que si las ganancias son cero para el sector competitivo (se hace este supuesto en (70)), las ganancias de ambos sectores en que hemos dividido la economía van a parar al sector monopolístico y, dado que este avanza su tasa a la tasa máxima, trae como consecuencia que todo el excedente (tasa máxima=excedente relativo) va a parar a las ganancias, incluido la parte del excedente que se repartía como salarios. Una vez más podemos ver en este modelo de dos sectores (monopolístico y no monopolístico) lo nefasto que puede llegar a ser el mercado si se permitiera una actuación de acaparamiento de todo el excedente. A diferencia de los modelos marginalistas, aquí no hay justificación de ese acaparamiento por supuestas productividades del capital ni asignación (eficiente o no) de los recursos, porque en este modelo de inspiración esrafiana las variaciones de las variables monetarias (precios, salarios y ganancias) no son necesariamente causa y/o efecto de cambio en las variables físicas (trabajo, medios, productos 
finales). Estas últimas son datos de la realidad. No es que no cuenten, es que se hace el supuesto (implícito) de comportamiento de que los que deciden en las empresas afrontan sus decisiones moviendo las variables monetarias en la medida que la circunstancias (el mercado, las leyes, las instituciones, las zonas geográficas) se lo permiten. El modelo no impide que muevan también en sus decisiones empresariales las variables físicas aludidas, pero el modelo lo toma como un dato, sin establecer relaciones de causa y efecto entre variables físicas y monetarias. En cualquier caso y en el caso hipotético de que se movieran las variables físicas medios, productos e inputs de trabajo, sucedería que en la frontera salario-ganancia que hemos estado discutiendo se produciría un desplazamiento de toda la frontera, bien hacia la derecha o a la izquierda. No necesariamente es incompatible con introducir en un modelo esrafiano funciones que intenten relacionar variables físicas y monetarias mediante estas relaciones causa y efecto, pero siempre que ello no lleve a disminuir los grados de libertad del sistema (modelo) de tal forma que salarios y ganancias pierdan entre sí su grado de libertad ${ }^{38}$. El reparto entre ambos depende de la lucha de clases (o en términos no marxistas, tensiones sociales). Lo que marcan los modelos esrafianos -o deben marcarlo si se reputan como tales- es el reparto del excedente y de sus límites, pero en ningún caso intervienen en la decisiones de reparto y asignación de recursos supuestas productividades marginales ni no marginales. Estas productividades lo que marcan son un límite para el excedente. En otras palabras, influyen en el tamaño de la tarta, no en su reparto. Creo que todo lo anterior es acorde con el espíritu esrafiano expresado en Producción de mercancías por medio de mercancías. Al menos esa es mi visión del mundo y la teoría abierta genialmente por el gran economista italiano.

Acabamos este artículo con la siguiente consideración. Dado que las funciones (52) y (53) son simétricas respecto a las tasas de ganancia (también lo son respecto a las tasas de salario), la tasa de ganancia media $\boldsymbol{g}_{\mathrm{A}}$ del sector competitivo puede expresarse como dependiente de la tasa del no competitivo $g_{\mathrm{B}}$ :

$$
g_{A}=\frac{\left[\left[1-w_{B} L_{B} I-w_{A} L_{A} I\right] g_{m B}-\left[1+w_{B} g_{m B} L_{B} I-w_{A} L_{A} I\right] g_{B}\right] g_{m A}}{\left[1-w_{B} L_{B} I+w_{A} g_{m A} L_{A} I\right] g_{m B}-\left[1+w_{B} g_{m B} L_{B} I+w_{A} g_{m A} L_{A} I\right] g_{B}}
$$

A diferencia de cuando teníamos las ganancias no competitivas de forma dependiente en (64) de las competitivas a las que hacíamos tender a cero (criterio económico y no meramente formal), aquí el criterio económico es el de que las no competitivas $\boldsymbol{g}_{\mathrm{B}}$ tiendan a la tasa de ganancia máxima $\boldsymbol{g}_{\mathrm{mB}}$. Pues bien, si empleamos ese criterio en (72) obtenemos la expresión:

\footnotetext{
${ }^{38}$ Así por ejemplo, Kurz en su Theory of Production trabaja en gran parte con modelos de su obra de origen esrafiano y utiliza las técnicas de programación lineal para optimizar variables, al igual que el modelo de Von Neumann, y no por ello traiciona el espíritu de la obra del turinés. Incluso en el capítulo de Sraffa sobre elección de técnicas (métodos de producción) hay optimización en la medida que se construyen dos (podrían ser más) fronteras salarios-ganancia que permite pasar al empresario que decide entre ambas técnicas (ambas fronteras) para que, con el mismo salario, pueda aumentar su ganancia. Eso está en Sraffa de hoz y coz.
} 


$$
\lim d e g_{A}\left(g_{B} \rightarrow g_{m b}\right) \rightarrow g_{m A}
$$

Es decir, si los empresarios del sector no competitivo llevan su tasa de ganancia $g_{\mathrm{B}}$ a la tasa máxima $g_{\mathrm{mB}}$, jno por ello la tasa de ganancia del competitivo $\boldsymbol{g}_{\mathrm{A}}$ disminuye, sino que tiende a su vez a su tasa máxima $\boldsymbol{g}_{\mathrm{mA}}$ ! La razón económica de esta paradoja es la siguiente: cuando estábamos en el primer modelo de este epígrafe donde un sector vendía al otro pero no le compraba, se producía una asimetría entre ambos en lo que respecta a las tasas de ganancia y, por ende, a sus precios de venta, puesto que una influía en el precio del productos final del otro pero no al revés (del sector vendedor al comprador, pero no al revés). En este modelo en el que estamos, ambos sectores se venden y compran mutuamente, por lo que un aumento de la tasa de ganancia en el otro sector influye en los precios del propio y, por consiguiente, en los costes del sector que compra sus productos finales; pero a su vez aumentan los ingresos por lo que respecta a los productos que vende al otro sector. El resultado final de esta escalada es que ambos sectores hacen tender sus ganancias hasta sus límites máximos, acaparando con ello los excedentes relativos. Pero si eso ocurriera porque la correlación de fuerzas entre empresarios y trabajadores fuera tan desfavorable a estos últimos tendría dos efectos indeseables: 1) los precios tenderían al infinito tal y como expuso Sraffa en el apéndice $\boldsymbol{B}$ sobre las mercancías que se auto-reemplazan (autoreproducen); 2) los salarios tenderían a cero por el acaparamiento del excedente antes señalado. Con este modelo la economía mantiene una tensión entre las decisiones de maximización de la economía de los sectores no competitivos y la lucha de los asalariados a mantener sus salarios. En el marginalismo se da una respuesta tecnológica -aunque descabellada e irrealde una supuesta retribución de los factores sobre la base de la productividad marginal; aquí, en este modelo de inspiración esrafiana y con la disección de sectores llevada a cabo por Pasinetti, no existe ese criterio productivista porque el reparto del excedente no depende este criterio; tampoco hay como solución final una situación de equilibrio y sí muchas situaciones intermedias entre los salarios y ganancias, entre asalariados y empresarios, teniendo ambos el excedente relativo como sus máximos posibles.

\section{Conclusiones}

Dado lo escueto del análisis anterior y su fuerte carga matemática en cuanto a la extensión, las conclusiones se han obtenido sin dificultad a lo largo del artículo. No obstante, alguna se puede indicar de carácter general: 1) Al abandonar la teoría de los mercados de origen marginalista (la caja de herramientas de Joan Robinson) se ha visto la dificultad de compatibilizar una teoría del mercado parcial a lo Marshall con la necesidad de atender a dos amos en esa nueva hipotética teoría del mercado de origen esrafiano: a) por un lado atender a las características de cada mercado y cómo se configura (de competitivo a monopolístico y situaciones intermedias) y por otro tener en cuenta que tanto rentas (ganancias y salarios) como precios han de permitir la 
reproducción del sistema en un modelo esrafiano. Esto lo diferencia de las teorías del mercado de origen marshalliano que excluyen esa necesidad mediante la cláusula caeteris paribus, es decir, al examinar cada mercado o empresa aisladamente, sin relación con el conjunto; 2) A pesar de lo anterior, hemos podido establecer unas relaciones entre salarios (masas o tasas unitarias), ganancias (ídem), ganancias máximas (ídem) y precios, que son no triviales y, en algunos casos, contrarias a las que surgen del marginalismo, bien sea del derivado del estudio de los mercados antes aludidos, del equilibrio general walrasiano o de modelos actuales; 3) Estas conclusiones a veces son análogas a las que surgen de los modelos de origen keynesiano/kaleckiano, pero sin el problema de agregación de estos; 4) La relación que se establece entre los dos tipos de ganancias (competitivas y no competitivas) resulta ser sorprendentemente lineal, a pesar de la complejidad del modelo y más aún cuando se incorpora la demanda. Ello es así porque: a) las rentas no se forman por variaciones en el margen de ninguna variable, b) porque no se introduce ninguna función de producción que rompa la linealidad entre producción y medios de producción, c) porque lo anterior permite mantener un grado de libertad entre rentas, tantas veces se dividan éstas entre sus posibles formas; 5) La variable de comportamiento del mercado que aquí se propone no está exenta de problemas. Se caracterizan los mercados competitivos como aquellos cuya tasa de ganancia tiende a cero. No es este un criterio distinto del marginalista. La diferencia de este que se propone -y que tiene como origen y consecuencia el reparto de rentas (entre tipos de ganancias y entre éstas y salarios) - y el marginalista es que este hace girar los ajustes de producción a la relación entre cantidad producida y costes marginales que de ello se deriva y la asignación de factores entre cantidad de factor y sus productividades marginales. No tengo dudas que la realidad irá siempre del lado de un modelo del mercado que tenga como consecuencia un reparto de rentas más que una reasignación de recursos a la manera marginalista. Sin embargo, el criterio monopolístico de obtención de la ganancia que aquí se propone sí es más problemático, aunque también mucho más realista. Se propone que una mercancía (en su caso sector o empresa) ha llegado a su techo máximo de acaparación de renta si sus ganancias llegaran a las tasas máximas de ganancias $^{39}$ de origen esrafiano. Pero ese extremo es inaceptable porque supondría la eliminación del salario. Ha de entenderse más como una posibilidad extrema, deseada por los empresarios individualmente, pero nefasta para el conjunto de la economía si se consiguiera alcanzar. Con ello se evidencia la gran contradicción del capitalismo, que está en las antípodas de la ingenua mano invisible smithiana. Por esta razón, por este reflejo henchido de realismo es por lo que ha mantenido el criterio a pesar de su problemática; 6) El modelo, a pesar de que no nos salimos de la reproducción al incorporar la demanda, obliga a una actuación de política económica (inversiones públicas, gasto público, tributos) si se quiere mantener el equilibrio del sistema, es decir, para evitar, ciclos y crisis, a diferencia de los modelos aceptados de equilibrio general. La realidad es la mejor juez para aceptar unos modelos $u$ otros. Desde luego lo que pasa actualmente y la historia de los ciclos y crisis han pisoteado intelectualmente los modelos ortodoxos normalmente aceptados de la economía. Este que se ha presentado, a pesar de su esquematismo, es un

\footnotetext{
${ }^{39}$ Tasa máxima de la producción conjunta (como la del modelo que se propone) equivalente a la razón-patrón de la producción simple.
} 
esbozo de modelo alternativo de inspiración esrafiana. Para las conclusiones de la incorporación del paradigma neoclásico sobre la relación capital/trabajo nos remitimos al último epígrafe; 7) En el epígrafe último inspirado en los criterios Sraffa-Pasinetti de división de sectores hemos comprobado que en cuanto se generaliza a un modelo donde ambos sectores (competitivo y no competitivo) comercian entre sí, no se puede establecer con seguridad una frontera monótona decreciente entre salarios y ganancias porque ello va a depender de las variables que intervienen en el modelo, constituyendo una refutación más de la teoría del capital neoclásica.

Un resultado interesante y no evidente cuando comenzábamos este epígrafe es que si en (70) el sector monopolístico pudiera aumentar las ganancias hasta llegar a la tasa máxima ocurriría que no sólo se verían afectados los salarios de este sector, sino que también se verían reducidos a ceros los del sector competitivo (en este esquema particular se han supuesto iguales). La razón económica es que si las ganancias son cero para el sector competitivo (se hace este supuesto en (70)), las ganancias de ambos sectores en que hemos dividido la economía van a para al sector monopolístico y, dado que este, al avanzar su tasa a la tasa máxima, trae como consecuencia que todo el excedente (tasa máxima=excedente relativo) va a parar a las ganancias, incluido la parte del excedente que se repartía como salarios. Una vez más podemos ver en este modelo de mercado de dos sectores (monopolístico y no monopolístico) lo nefasto que puede llegar a ser el mercado si permitiera una actuación de acaparamiento de todo el excedente. A diferencia de los modelos marginalistas, aquí no hay justificación de ese acaparamiento por supuestas productividades del capital ni asignación (eficiente o no) de los recursos porque en este modelo de inspiración esrafiana las variaciones de las variables monetarias (precios, salarios y ganancias) no son causas ni efecto de cambio en las variables físicas (trabajo, medios, productos finales). Estas últimas son datos de la realidad. No es que no cuenten, es que se hace el supuesto (implícito) de comportamiento de que los que deciden en las empresas afrontan sus decisiones moviendo las variables monetarias en la medida que la circunstancias (el mercado, las leyes, las instituciones, las zonas geográficas) se lo permiten. El modelo no impide que muevan también en sus decisiones empresariales las variables físicas aludidas, pero el modelo lo toma como un dato, sin establecer relaciones de causa y efecto entre variables físicas y monetarias. No necesariamente es incompatible introducir en un modelo esrafiano funciones que intenten relacionar variables físicas y monetarias mediante estas relaciones causa y efecto siempre que ello no lleve a disminuir los grados de libertad del sistema (modelo) de tal forma que salarios y ganancias pierdan entre sí su grado de libertad ${ }^{40}$. El reparto entre ambos depende de la lucha de clases (o en términos no marxistas, tensiones sociales). Lo que marcan los modelos esrafianos y deben marcarlo si se

\footnotetext{
${ }^{40}$ Así por ejemplo, Kurz en su Theory of Production trabaja en gran parte con modelos de su obra de origen esrafiano y utiliza las técnicas de programación lineal para optimizar variables, al igual que el modelo de Von Newman, y no por ello traiciona el espíritu de la obra del turinés. Incluso en el capítulo de Sraffa sobre elección de técnicas (métodos de producción) hay optimización en la medida que se construyen dos (podrían ser más) fronteras salarios-ganancia que permite pasar al empresario que decide entre ambas ténicas (ambas fronteras) para que con el mismo salario pueda aumentar su ganancia. Eso está en Sraffa de hoz y coz.
} 
reputan como tales es el reparto del excedente y de sus límites, pero en ningún caso intervienen en la decisiones de reparto y asignación de recursos supuestas productividades marginales ni no marginales. Estas productividades lo que marcan son un límite para el excedente. En otras palabras, influyen en el tamaño de la tarta no en su reparto. Creo que todo lo anterior es acorde con el espíritu esrafiano expresado en Producción de mercancías por medio de mercancías. Al menos esa es mi visión del mundo y la teoría abierta genialmente por el gran economista italiano.

\section{Apéndice: significado de la tasa máxima de ganancia}

Veamos una forma de abordar la estimación de las tasas máximas de ganancia es como sigue. A partir de la ecuación de definición del sistema ${ }^{41}$ :

$$
P Y=[L W+P X](I+G)
$$

obtenemos la ecuación de precios mediante simples manipulaciones matriciales:

$$
P=L W\left(I_{d}+G\right)[Y-X(I+G)]^{-1}
$$

Por otro, tenemos la ecuación de precios derivada de hacer cero las tasas de salario también en la ecuación de definición del sistema:

$$
P=L W\left(I_{d}+G\right)\left[(B-G)^{-1} X^{-1}\right]
$$

donde la novedad es $\boldsymbol{B}$ que es la matriz de tasas máximas de ganancia. A simple vista se puede observar que se puede establecer una relación lineal entre la resultante de la matriz entre corchetes de (A1) y (A2). Por ello se puede conjeturar la siguiente relación:

$$
\left[Y-X\left(I_{d}+G\right)\right]^{-1}=\left[(B-G)^{-1} X^{-1}\right] F
$$

siendo $\boldsymbol{F}$ una matriz diagonal que podemos calcular porque tenemos $\boldsymbol{n}$ ecuaciones y $\boldsymbol{n}$ incógnitas. Si ahora hacemos las tasas de ganancia igual a cero, la nueva relación de (A4) queda:

$$
[Y-X]^{-1}=B^{-1} X^{-1} H
$$

${ }^{41}$ En este apéndice las matrices son todas cuadradas, a diferencia del texto principal. No obstante ello no cambia las consideraciones sobre el significado de las tasas máximas de ganancias que aquí se establecen. 
siendo a su vez $\boldsymbol{H}$ la nueva matriz diagonal que hace posible (A5). Y premultiplicando (A5) por $\boldsymbol{B}$ y post-multiplicando por ( $\boldsymbol{Y}-\boldsymbol{X})$ sale:

$$
B=X^{-1} H(Y-X)
$$

Esta última ecuación expresa claramente que las tasas máximas de ganancias son una medida del excedente (relativo) porque $\boldsymbol{Y}$ - $\boldsymbol{X}$ es la diferencia entre productos finales y medios de producción necesarios para llegar a los primeros.

También (A6) puede escribirse como:

$$
B=X^{-1}(Y-X) K=X^{-1} Y M
$$

Y en términos de tasa unitaria:

$$
b=X^{-1}(Y-X) K I=f\left(X^{-1}(Y-X)\right)
$$

Con $\boldsymbol{M}$ también como matriz diagonal y factor de proporcionalidad que permite estimar las tasas máximas de ganancia. La ecuación (A7) nos dice que las tasas máximas de ganancia son proporcionales a una media ponderada del excedente relativo que representa $\boldsymbol{X}^{-1} \boldsymbol{Y}$.

Visto así, hemos demostrado el carácter dual de las tasas máximas de ganancias: son a la vez una medida del excedente y una manera (un índice) de valorar la productividad del sistema. No por ello hay que caer en un marginalismo porque no hay productividades marginales, es decir, variaciones en el margen. Todo esto permite partir y acercarse a la realidad con la naturalidad de la que está ausente el marginalismo. Veamos sus ventajas: 1) En efecto, los elementos de las matrices de productos $\boldsymbol{Y}$ y de medios $\boldsymbol{X}$ podríamos muy bien obtenerlas de la realidad; 2) No es necesario hacer explícito una función de producción, ni siquiera en el caso de que aquéllas no acabaran con el grado de libertad entre salarios y ganancias, cosa imprescindible para ser considerado un modelo al menos de origen esrafiano. Esta no es incompatible con el esquema esrafiano, pero no es necesaria su explicitación porque en el modelo intersectorial esrafiano, tanto productos como medios, son tomados de la realidad. Con ello Sraffa huyó como de la peste de las consideraciones sobre rendimientos constantes que le hacía su amigo y mentor Keynes; 3) Las tasas máximas de ganancia acaparan todo el excedente y lo que queda es lo que permite la reproducción del sistema en sus mismas condiciones. 


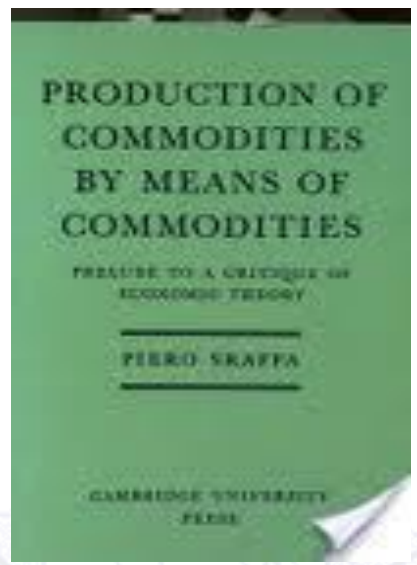

\section{Bibliografía}

Afriat, S.: "Sraffa's Prices", Universitá degli Studi di Siena, quaderni 474. www.econ-pol.unisi.it/quaderni/474.pdf

Ahijado, M.: "Distribución, precios de producción y crecimiento", 1982, Centro de Estudios Universitarios Ramón Areces.

Ahijado, M.: "Piero Sraffa: notas para una biografía intelectual", 1985, Centro de Estudios Universitarios Ramón Areces.

Barceló, A. y Sánchez, J.: "Teoría económica de los bienes autorreproducibles", Edit. Oikos-Tau, 1988.

Blanchard. O.: "Macroeconomía", edit. Prentice-Hall, 2000 (Macroeconomics, 2000).

Bour, Enrique A.: "Marx y la teoría económica moderna", 2007 http://www.aaep.org.ar/anales/works/works2007/bour.pdf

Caballero, A. y Lluch, E.: "Sraffa en España", Investigaciones Económicas (2a época, vol. X, n.ำ2), 1986.

Dobb, M.: "Teoría del valor y de la distribución desde Adam Smith", edit. Siglo $\mathrm{XXI}$ editores. 
Desai, M.: "Marxian Economic Theory", 1974 [Lecciones de teoría económica marxista, 1977, edit. Siglo XXI].

Dobb, M.: "The Sraffa system and the critique of neoclassical theory of distribution", 1970.

Estrin, S. y Laidler, D: "Introduction microeconomics".

Fiorito, Alejandro: "La implosión de la economía neoclásica". Está en la red: www.geocities.com/aportexxi/sraffa12.pdf

Foncerrada, Luis Antonio: "Sraffa y Böhm-Bawerk". Está en la red: http://www.economia.unam.mx/secss/docs/tesisfe/FoncerradaPLA/tesis.pdf

García, N.E.: "La crisis de la macroeconomía”, edit. Marcial Pons, 2010.

Garegnani, P.: "El capital en la teoría de la distribución", 1982, ed. Oikos-Tau ("Il capitale nelle teorie delladistribuzone", 1982)

Garegnani, P.: "Heterogeneous Capital, The Production Function and the Theory Distribuction", 1970

Garegnani, P.:"Professor Samuelson on Sraffa and the Clasical Economists", The European Journal of the History of Economic Thought, 2007

Gehrke, Ch.y Kurz, D.: "Sraffa on von Bortkiewicz". Está en la red: http://www.newschool.edu/cepa/events/papers/050509_Bortkiewicz.pdf

Harcourt, G.C.: "Teoría del Capital" (Some Cambridge controversies in the theory of capital, 1975), apéndice al cap. 4, 1975, edit. Oikos-tau.

Heahtfield, D. F.: "Productions functions".

Keynes, J.M.: "Teoría General de la Ocupación, el Interés y el Dinero", FCE, 1992 (The General Theory of Employement, the Interest and Money, 1936).

Korsch, Karl; "Karl Marx”, 1975, traducción de Manuel Sacristán, edit. Ariel.

Kurz, Pasinetti, Salvador y otros: "Piero Sraffa: The Man and the Scholar", Routledge, 2008.

Kurz y Salvadori: "Sraffa and the mathematicians: Frank Ramsey and Alister Watson", en "Piero Sraffa's Political Economy, edit Routledge,

Kurz, D. Heinz; "Critical Essays on Piero Sraffa's Legacy in Economics", 2000, Cambrigde University Press.

Kurz, D. Heinz; Salvadori, Neri; “Theory of Production”, 1997. 
Lange, O., Taylor, F. M.: "On the Economic Theory of Socialism, 1938 [Sobre la teoría económica del socialismo, 1971, edit. Ariel]

Marsahll, Alfred: "Principios de Economía, Fundación ICO, 2005 [Principles of Economy, 1890]

Marx, Carlos: "El método en la Economía Política", 1974, Ediciones Grijalbo, S.A.

Marx, Carlos: "El Capital”, en el FCE, traducción de Wenceslao Roces.

Meade, J.: “A neo Classical Theory of Economic Growth”, 1961.

Meek, R.: “Mr. Sraffa's Rehabilitationof Classical Economics”, 1961.

Mendoza, Gabriel: "La transformación de valores en precios de producción", 1997

http://www.izt.uam.mx/economiatyp/numeros/numeros/10/articulos_PDF/10_2_ La_transformacion.pdf

Mora Plaza, A.: "Aspectos de la economía de Sraffa", revista: Nómadas, n. 23, U. Complutense de Madrid, enlace:

http://www.ucm.es/info/nomadas/23/antoniomora.pdf

Mora Plaza, A.: "Notas sobre la producción simple y conjunta a consecuencia de Sraffa”: http://redalyc.uaemex.mx/pdf/181/18112179020.pdf;

Mora Plaza, A.: "Sobre la transformación de valores a precios": http://www.eumed.net/ce/2009b/amp2.htm

http://revistas.ucm.es/cps/15786730/articulos/NOMA1010140379A.PDF

Mora Plaza, A.: "Notas sobre el teorema fundamental marxiano"

http://www.eumed.net/ce/2009b/amp.htm

http://econpapers.repec.org/article/ervcontri/y_3a2009_3ai_3a2009-

10_3a22.htm

Mora Plaza, A: "Descifrando a Sraffa”, Editorial Académica Española, 2011.

Morhisima, M.: "La teoría económica de Marx" (Marx’s Economics, 1973), 1977, pág. 15, edit. Tecnos.

Moseley, F.: "El método lógico y el problema de la transformación". http://www.azc.uam.mx/publicaciones/etp/num7/a8.htm

Murga, Gustavo: "Piero Sraffa".

http://marxismo.cl/portal/index.php?option=com_content\&task=view\&id=100\&lte mid $=1$

Neri, Salvador: "Besicovitch, Sraffa and the existence of Standard Commodity", 2010:

http://host.uniroma3.it/eventi/sraffaconference2010/abstracts/pp_salvadori.pdf 
Nuti, D.: "Capitalism, Socialism and Sleady Growth", 1970.

Okishio, N.: "A mathematical note on marxian theorems", 1963.

Pasinetti. L.: "Critical of the neoclassical theory of growth and distribution". Está en la red: http://www.unicatt.it/docenti/pasinetti/pdf_files/Treccani.pdf

Pasinetti, L.: "Structural Change and Economic Growth: a theoretical essay on the dynamics of Wealth of Nations", 1981, Cambridge University Press.

Pasinetti, L.: "Rate of profit and income distribution in relation to the rate of economic growth", 1961/2.

Pasinetti, L.: "Switches of technique and the rate of return in Capital Theory", 1969.

Pasinetti, L.: "Crecimiento económico y distribución de la renta" ("Growth and Income Distribution", 1974), 1978, Alianza Editorial.

Pasinetti, L.: "Lecciones de teoría de la producción" ("Lezioni di teoria della produzioni”, 1975), 1983, FCE.

Peris i Ferrando, J.E: "Análisis de la resolubilidad de modelos lineales de producción conjunta", 1987, en internet: http://rua.ua.es/dspace/bitstream/10045/3829/1/Peris\%20Ferrando,\%20Josep.p df

Potier, J.P.: "Piero Sraffa", 1994, edicions Alfons Magnànim.

Ricardo, D.: "Principios de Economía Política y Tributación" (On the Principles of Political Economy and Taxation,), 1973, F.C.E.

Robinson, J.: “Ensayos críticos”, 1984, Ediciones Orbis.

Roncaglia, Alessandro: "Piero Sraffa”, Edit. Palgrave MacMillan, 2009.

Roncaglia, Alessandro: "La riqueza de las ideas", Prensas Universitarias de Zaragoza, 2009 ("The Wealth of Ideas. A History of Economic Thought", Cambridge University Press, 2005).

Roncaglia, Alessandro: "Sraffa and the Theory of Prices", 1978 [Sraffa e la teoria dei prezzi, 1975]

Samuelson, Paul: "Understanding the Marxian notion of Exploitation”, 1971.

Sánchez Choliz, Julio: “La razón-patrón de Sraffa y el cambio técnico”, 1989, Investigaciones Económicas, 2ª época, Vol. XIII.

ftp://ftp.funep.es/InvEcon/paperArchive/Ene1989/v13i1a7.pdf 
Sargent, T.J.: "Teoría macroeconómica" (Macroeconomic Theory, 1979), 1988, Antoni Bosch editor.

Schefold, Bertram: "Mr. Sraffa on Joint Production", 1971

Schumpeter, J. A.: "Historia del Análisis Económico" (History of Economic Analisis, 1954), 1971, Ediciones Ariel.

Segura, J.: "Análisis microeconómico", pág. 88, 2004, Alianza editorial Tecnos.

Serrano, Franklin: "Histérisis, Dinámica inflacionaria y el Supermultiplicador Sraffiano", 2006: $\quad$ http://www.elgermen.com.ar/wordpress/wpcontent/uploads/Serrano-F-Hist\%C3\%A9resis-Din\%C3\%A1mica-Inflacionaria-yel-Supermultiplicador-Sraffiano.pdf

Steedman, I.: "Marx, Sraffa y el problema de la transformación" (Marx after Sraffa, 1977), 1985, F.C.E.

Subiza Martínez, B.: "Juegos matriciales y su aplicación a la teoría PerronFrobenius", U. de Alicante; http://www.ine.es/revistas/estaespa/112_3.pdf

Solow, R.: "The interest rate and transition between techniques", 1967.

Sraffa, Piero: "Produción de mercancías por medio de mercancías" (Production of commodities by means commodities, 1960), 1975, Oikos-Tau.

Ricardo, D.: "Principios de Economía Política y Tributación" (On the Principles of Political Economy and Taxation,), 1973, F.C.E.

Vegara, J. M.: "Economía política y modelos multisectoriales",1979, edit. Tecnos.

Varios: "Matemáticas avanzadas aplicadas a la Economía”, UNED, 2001.

Varios: "The Keynesian Multiplier", edit. Routledge Frontiers of Political Economy, 2008

Woods, J. E.: "The Production of Commodities. An Introduction to Sraffa", edit. MacMillan, 1990.

$$
\begin{gathered}
p_{j} y_{j}=w l_{j}+(1+r) \sum_{i=1}^{n} p_{i} x_{i j} \text { desde } j=1 \text { a } j=\mathrm{n} \\
p_{j} y_{j}=(1+R) \sum_{i=1}^{n} p_{i} x_{i j} \text { desde } j=1 \text { a } j=\mathrm{n} \\
\sum_{j=1}^{n} p_{j} y_{j}-\sum_{j=1}^{n} \sum_{j=1}^{n} p_{i} x_{i j}=1 \\
\sum_{1}^{n} l_{j}=1
\end{gathered}
$$

